\title{
Some Guidelines for Implementing Error Compensation on Machine Tools
}

M. A. Donmez

K. W. Yee

B. Damazo

U.S. DEPARTMENT OF COMMERCE Technology Administration National Institute of Standards and Technology Automated Production Technolgy Division Manufacturing Engineering Laboratory Gaithersburg, MD 20899 



\title{
Some Guidelines for Implementing Error \\ Compensation on \\ Machine Tools
}

\author{
M. A. Donmez \\ K. W. Yee \\ B. Damazo
}

\section{U.S. DEPARTMENT OF COMMERCE Technology Administration National Institute of Standards and Technology Automated Production Technolgy Division Manufacturing Engineering Laboratory Gaithersburg, MD 20899}

August 1993

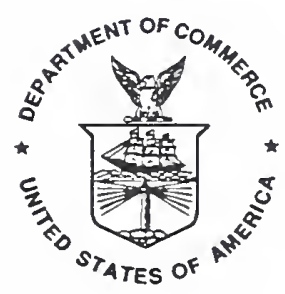

U.S. DEPARTMENT OF COMMERCE Ronald H. Brown, Secretary TECHNOLOGY ADMINISTRATION Mary L Good, Under Secretary for Technology NATIONAL INSTITUTE OF STANDARDS 


\section{TABLE OF CONTENTS}

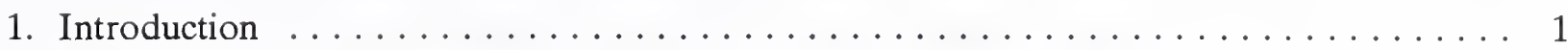

2. A Mathematical Model for Machine Tool Errors . . . . . . . . . . . . . . . . 2

2.1 Homogeneous Transformations - Background . . . . . . . . . . . . 3

2.2 Homogeneous Coordinate Representation of Machine Slides . . . . . . . . . . 4

2.3 The Model for Machine Tools . . . . . . . . . . . . . . . . . . . . . 8

3. Machine Characterization .......................... 21

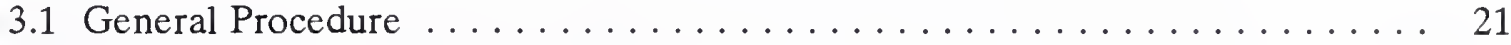

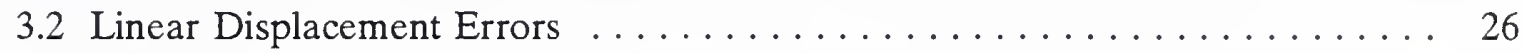

3.3 Angular Errors . . . . . . . . . . . . . . . . . . . 26

3.4 Straightness-Parallelism-Orthogonality . . . . . . . . . . . . . . . . 29

3.5 Spindle Thermal Drift . . . . . . . . . . . . . . . . . . 40

3.6 Data Analysis . . . . . . . . . . . . . . . . . . . 43

4. Implementing Error Compensation $\ldots \ldots \ldots \ldots \ldots \ldots \ldots \ldots \ldots \ldots \ldots \ldots \ldots \ldots \ldots$

4.1 Potential Problems Limiting Implementation . . . . . . . . . . . . . . 46

4.1 .1 Case Study Using Hardware Modifications . . . . . . . . . . . . . . . 47

4.1 .2 Case Study Using Software Modifications . . . . . . . . . . . . . 48

4.2 A Generic Approach . . . . . . . . . . . . . . . . . . . 50

4.2 .1 RTEC Operationg Principles . . . . . . . . . . . . 50

4.2.2 The Way of the Future .................... 52

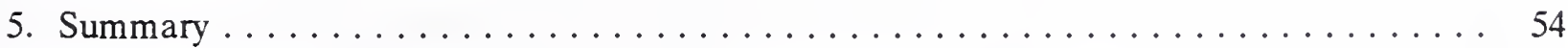

6. References $\ldots \ldots \ldots \ldots \ldots \ldots \ldots \ldots \ldots \ldots \ldots \ldots \ldots \ldots \ldots$ 


\section{LIST OF FIGURES}

Figure 2.1 Six degrees of freedom error motion of a typical machine slide $\ldots \ldots \ldots \ldots 5$

Figure 2.2 Coordinate frame assignments on a two-axis turning center $\ldots \ldots \ldots \ldots \ldots$

Figure 2.3 The resultant error components at the tip of the cutting tool. (For single point

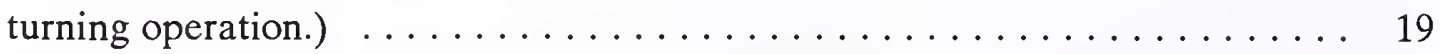

Figure 2.4 Schematic of $31 / 2$-axis fixed column vertical machine center $\ldots \ldots \ldots \ldots 20$

Figure 3.1 Temperature profile of the machine elements under continuous running

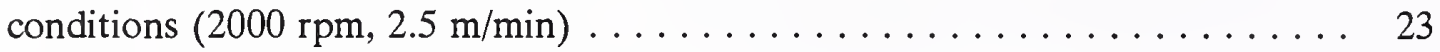

Figure 3.2 Temperature profile of the spindle under continuous running conditions (2000

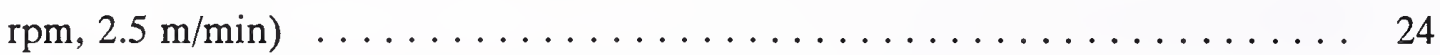

Figure 3.3 Vertical Machining Center Thermocouple locations for machine characteriza-

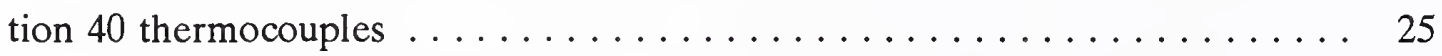

Figure 3.4 The laser interferometer set-up for $\mathrm{x}$ displacement error measurements . . . . 27

Figure 3.5 The laser interferometer set-up for $z$ displacement error measurements . . . . 28

Figure 3.6 The laser interfereometer set-up for $\mathrm{x}$ yaw error measurements . . . . . . . 30

Figure 3.7 The laser interferometer set-up for $z$ yaw error measurements . . . . . . . . 31

Figure 3.8 The set-up for the $\mathrm{x}$ straightness of the $\mathrm{z}$ motion and the parallelism measurements (probes are at the beginning of the $\mathrm{z}$ travel. . . . . . . 32

Figure 3.9 The schematic of the $x$ straightness of the $z$ motion measurement technique $\ldots 34$

Figure 3.10 The schematic for the probe locations to calculate the parallelism error $\ldots . .36$

Figure 3.11 The set-up for the $\mathrm{z}$ straightness of the $\mathrm{x}$ motion and the orthogonality

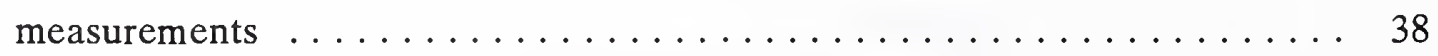

Figure 3.12 The schematic for calculating the $\mathrm{z}$ straightness of the $\mathrm{x}$ motion $\ldots \ldots \ldots . .39$

Figure 3.13 The set-up for the radial spindle drift measurements $\ldots \ldots \ldots \ldots \ldots \ldots .41$

Figure 3.14 The set-up for the axial spindle drift measurements $\ldots \ldots \ldots \ldots \ldots \ldots .42$

Figure 3.15 Thermocouple locations during the spindle drift measurements . . . . . . . 44

Figure 4.1 Hardware specific error compensation $\ldots \ldots \ldots \ldots \ldots \ldots \ldots \ldots \ldots$

Figure 4.2 Software specific error compensation $\ldots \ldots \ldots \ldots \ldots \ldots \ldots \ldots \ldots \ldots . \ldots . \ldots$

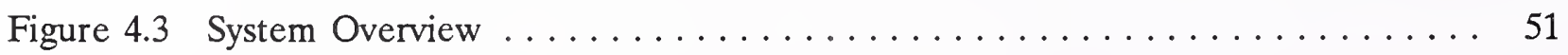

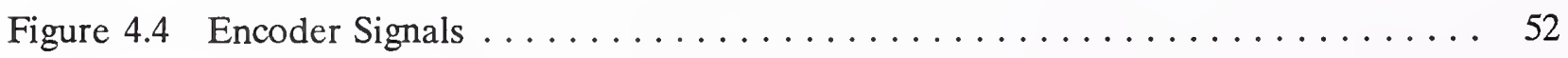




\section{Introduction}

In machining, the accuracy of the workpiece depends on the accuracy of the relative position between the cutting tool tip and the workpiece. Geometric and thermally-induced errors of the machine tools are the major contributing factors affecting the accuracy. Geometric errors are caused by the unwanted motions of machine elements, such as carriages, spindles, and work tables. These motions occur because of the geometric imperfections and the misalignments created during the assembly of the machine tool. Heat generated by the machine tool and the cutting process causes temperature elevations of the machine elements. Due to the complex geometry of the machine structure, concentrated heat sources, such as the drive motors, create thermal gradients along the machine structure. The spindle growth, the leadscrew, cutting tool and workpiece expansions, and the significant part of the machine structure deformation are the results of these temperature elevations and the gradients. These structural deformations in turn change the geometric error behavior of the machine tool resulting in the change in the relative position between the cutting tool tip and the workpiece.

In order to improve machine accuracy through real-time error compensation, machine geometric errors as well as thermally-induced errors have to be predicted and compensated [1]. The guidelines presented here are not meant to be definitive. They are based on our experience gained over the past several years with several different classes of machine tools. We believe that they are helpful for implementing error compensation to other classes of machine tools as well. The methodology described in this document requires the characterization of each individual error component of the machine-tool positioning elements, an error synthesis based on rigid body kinematics to obtain the resultant error at the cutting tool tip/workpiece interface [2], and a modification of the controller position servo loops through a hardware and/or software interface.

A mathematical model to calculate the resultant error vector from the individual error components was presented in Section 2. Machine characterization techniques to establish relationships between individual error components of each machine element and the temperature profile of the machine tool are described in Section 3. Some examples of real-time error compensation implementation are described in Section 4 of this report. 


\section{A Mathematical Model for Machine Tool Errors}

This section presents a generalized mathematical model for machine-tool errors. The model represents the positioning error of the cutting tool tip with respect to the workpiece as a combination of the individual machine component errors.

Machine tools are multidegree-of-freedom structures which consist of stacked slides and spindles. Each moving element of the machine tool has error motions in six degrees of freedom, three translations and three rotations. Therefore, depending on the number of axes (slides), a machine tool can have as many as 30 individual error components. Furthermore, the effect of the error of one slide may be amplified by the motion of another slide. On the other hand, the end effect of all these individual error components is a single translation and a rotation of the cutting tool around an arbitrary axis in space. Thus, for a single-point cutting operation, a simple translation is enough to compensate for the errors to achieve higher machining accuracy. When the orientation of the cutting tool is critical, additional rotational axes are required to compensate for these errors.

With the computing power of microprocessors, total software-based error compensation for machine tool errors (based on the mathematical error models) has become feasible. The mathematical error models created for this purpose should be easily transformed into a modular and maintainable software. Furthermore, these error models should be in a general format so as to be easily adapted to different machine tool configurations.

Based on these criteria, we present a generalized approach to develop the total error model for a machine tool, which systematically combines all the error components and yields an error vector. In developing this model, with the assumption of rigid body kinematics, we considered a machine tool-fixture-workpiece system as a chain of linkages and described the spatial relationships between these linkages using homogeneous coordinate transformation matrices. The model presented here can be applied to any machine tool or any type of positioning machines such as coordinate measuring machines or robots. 


\subsection{Homogeneous Transformations - Background}

In general, a homogeneous coordinate representation is defined as the representation of an $\mathrm{N}$ dimensional position vector by an $(\mathrm{N}+1)$-dimensional vector $[3,4]$. The $(\mathrm{N}+1)$ th component of this vector is called a scale factor. In a homogeneous coordinate representation, the actual components of an $\mathrm{N}$-dimensional vector can be found by dividing each component of the $(\mathrm{N}+1)$-dimensional vector by the scale factor. In a 3-dimensional space, the homogeneous coordinate representation of a vector $p=\left(p_{x}, p_{y}, p_{z}\right)^{t}$ is $p^{\prime}=\left(p_{x} p_{y}, p_{z}, 1\right)^{t}$, of which the scale factor is selected as unity.

A homogeneous coordinate transformation matrix in 3 -dimensional space is a $4 \times 4$ matrix. It is used to express a homogeneous coordinate vector in one coordinate system with respect to another coordinate system. Similarly, a homogeneous transformation matrix can be used to represent one coordinate system with respect to another or reference coordinate system. A homogeneous transformation matrix consists of four homogeneous coordinate vectors as shown below:

$$
T=\left[\begin{array}{cccc}
o_{1 x} & o_{2 x} & o_{3 x} & p_{x} \\
o_{1 y} & o_{2 y} & o_{3 y} & p_{y} \\
o_{1 z} & o_{2 z} & o_{3 z} & p_{z} \\
o_{1 s} & o_{2 s} & o_{3 s} & p_{s}
\end{array}\right]
$$

The vectors $o_{1}, o_{2}$, and $o_{3}$ describe the orientation (i.e., the directions of three unit vectors) of a coordinate frame with respect to another (or reference) coordinate frame. The vector $p$ is the position vector of the origin of the first coordinate frame with respect to the same (or reference) frame. Since vectors $o_{1}, o_{2}$, and $o_{3}$ are direction vectors with infinite length, their scale factors are zero. On the other hand, to express vector $p$ in its physical coordinates, the scale factor $p_{s}$ is set to unity. Thus, a general homogeneous transformation matrix with null perspective transformation is in the following form: 


$$
T=\left[\begin{array}{cccc}
o_{1 x} & o_{2 x} & o_{3 x} & p_{x} \\
o_{1 y} & o_{2 y} & o_{3 y} & p_{y} \\
o_{1 z} & o_{2 z} & o_{3 z} & p_{z} \\
0 & 0 & 0 & 1
\end{array}\right]
$$

With this matrix, it is possible to describe the relative rotation and translation between any two coordinate frames. If the coordinate frame is embedded in an object, then the above matrix describes the relative position and orientation of this object with respect to another object or coordinate frame in space. An important feature of homogeneous coordinate transformation matrices is that they can be multiplied in series to describe one object with respect to several different coordinate frames. For example, if ${ }^{R} T_{1}$ is the matrix which describes the position and orientation of Object 1 with respect to the reference, and ${ }^{1} T_{2}$ is the matrix which describes the position and orientation of Object 2 with respect to Object 1 , then the resultant matrix from the multiplication ${ }^{R} T_{1}{ }^{1} T_{2}$ describes the position and orientation of Object 2 with respect to the reference frame. This feature is very useful to describe structures which consist of several elements (or linkages) that are positioned with respect to each other. In order to simplify the notation, superscript $\mathrm{R}$ will be omitted from the rest of the equations.

\subsection{Homogeneous Coordinate Representation of Machine Slides}

A special case of representing the objects with respect to a reference coordinate system or another object can be applied to machine slides. A machine tool consists of several rigid links (slides) connected in series by prismatic joints. By assigning a coordinate frame to a slide and using a homogeneous transformation matrix, it is possible to describe the motion of a slide in a reference coordinate system. For example, consider a slide, as shown in Figure 2.1 (a), which moves along its $\mathrm{X}$-axis. The pure translation of such an ideal slide is described by a homogeneous transformation matrix in the following form: 

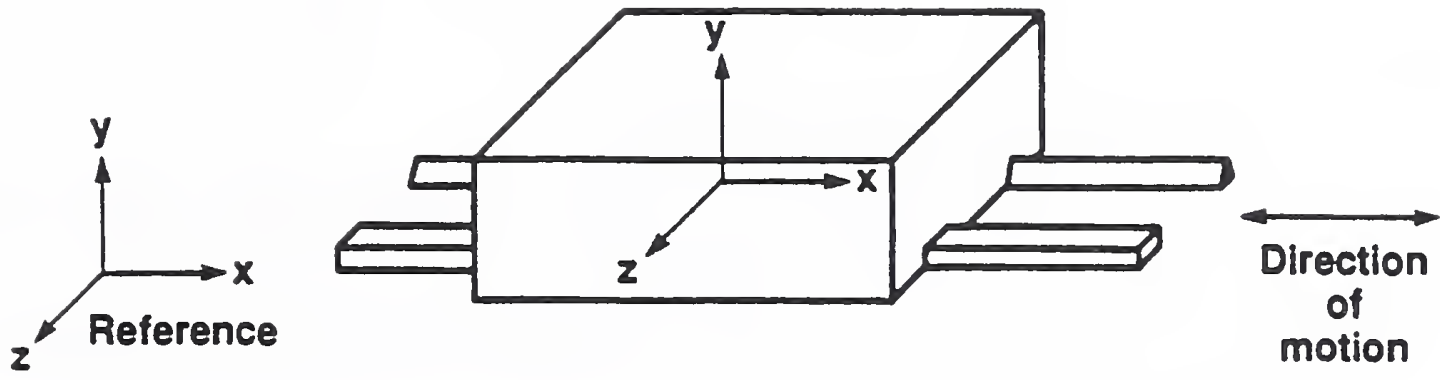

(a) Ideal slide

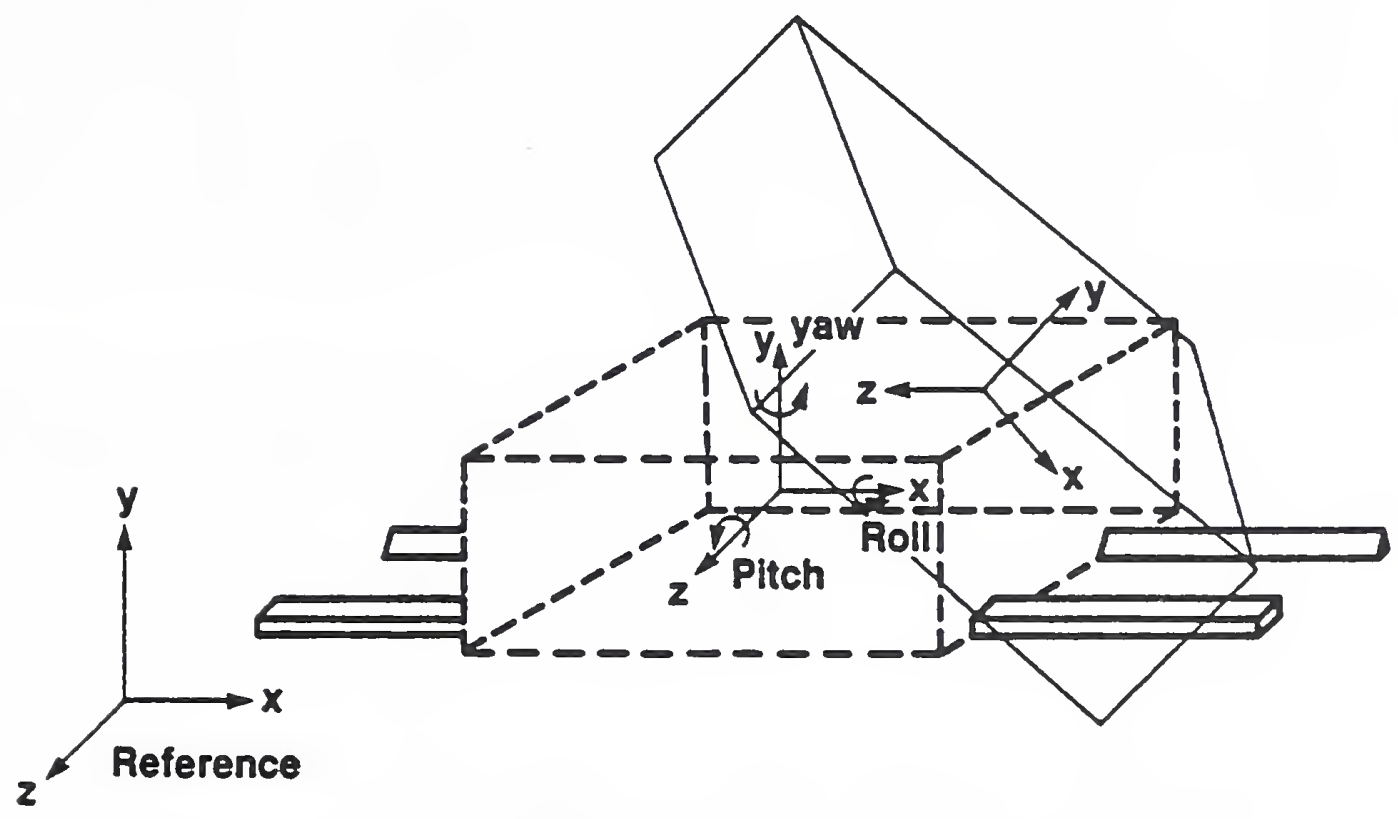

(b) Actual slide

Figure 2.1 Six degrees of freedom error motion of a typical machine slide. 


$$
T_{i}=\left[\begin{array}{llll}
1 & 0 & 0 & x \\
0 & 1 & 0 & Y \\
0 & 0 & 1 & Z \\
0 & 0 & 0 & 1
\end{array}\right]
$$

where,

$\mathrm{T}_{\mathrm{i}}$ is the description of ideal slide motion,

$Y$ and $Z$ are constant offsets of the origin of the slide coordinate system with respect to the reference coordinate system, and

$x$ is the variable position of the slide coordinate system origin with respect to the reference coordinate system.

In reality, in addition to its intended motion, any single-degree-of-freedom slide has error motions in six degrees of freedom [5]. Due to these unwanted motions, the actual position and the orientation of the slide coordinate frame is different from that of the ideal slide, as shown in Figure 2.1 (b). The total error motion of the slide can be considered as a combination of a rotation and a translation. The rotational error has three components, which correspond to the rotations about three orthogonal axes. These are defined as roll, pitch and yaw. The homogeneous transformation matrix, $E_{\text {rot }}$, which represents this total rotational error is given below with the assumption of small angular errors:

$$
E_{r o t}=\left[\begin{array}{cccc}
1 & -\varepsilon_{z} & \varepsilon_{y} & 0 \\
\varepsilon_{z} & 1 & -\varepsilon_{x} & 0 \\
-\varepsilon_{y} & \varepsilon_{x} & 1 & 0 \\
0 & 0 & 0 & 1
\end{array}\right]
$$

where,

$\epsilon_{x}$ is rotational error about x-axis (roll in Figure 2.1b),

$\epsilon_{y}$ is rotational error about y-axis (yaw in Figure 2.1b),

$\epsilon_{z}$ is rotational error about s-axis (pitch in Figure 2.1b). 
The total translational error matrix, $E_{\text {trans }}$, consists of three components which are translations along the three orthogonal axes:

$$
E_{\text {trans }}=\left[\begin{array}{cccc}
1 & 0 & 0 & \delta_{x} \\
0 & 1 & 0 & \delta_{y} \\
0 & 0 & 1 & \delta_{z} \\
0 & 0 & 0 & 1
\end{array}\right]
$$

where,

$\delta_{x}$ is the translational error along $x$-axis,

$\delta_{y}$ is the translational error along $y$-axis,

$\delta_{z}$ is the translational error along z-axis.

So, the total error matrix E (neglecting second order terms) is:

$$
\begin{aligned}
E & =E_{\text {rot }} E_{\text {trans }} \\
E & =\left[\begin{array}{cccc}
1 & -\varepsilon_{z} & \varepsilon_{y} & \delta_{x} \\
\varepsilon_{z} & 1 & -\varepsilon_{x} & \delta_{y} \\
-\varepsilon_{y} & \varepsilon_{x} & 1 & \delta_{z} \\
0 & 0 & 0 & 1
\end{array}\right]
\end{aligned}
$$

With this general error matrix, the actual position and orientation of the slide in the reference coordinate frame is found by the following equation: 


$$
\begin{aligned}
T_{a} & =T_{i}{ }^{i} E \\
& =\left[\begin{array}{llll}
1 & 0 & 0 & x \\
0 & 1 & 0 & Y \\
0 & 0 & 1 & Z \\
0 & 0 & 0 & 1
\end{array}\right]\left[\begin{array}{cccc}
1 & -\varepsilon_{z} & \varepsilon_{y} & \delta_{x} \\
\varepsilon_{z} & 1 & -\varepsilon_{x} & \delta_{y} \\
-\varepsilon_{y} & \varepsilon_{x} & 1 & \delta_{x} \\
0 & 0 & 0 & 1
\end{array}\right] \\
& =\left[\begin{array}{cccc}
1 & -\varepsilon_{z} & \varepsilon_{y} & \delta_{x}+x \\
\varepsilon_{z} & 1 & -\varepsilon_{x} & \delta_{y}+Y \\
-\varepsilon_{y} & \varepsilon_{x} & 1 & \delta_{z}+Z \\
0 & 0 & 0 & 1
\end{array}\right]
\end{aligned}
$$

where $T_{a}$ is the description of actual slide motion.

This type of homogeneous transformation matrix representation will be used for all the elements of the machine tool in the analysis that follows.

\subsection{The Model for Machine Tools}

Since a machine tool can be considered as a chain of linkages, an approach similar to the Denavit-Hartenberg approach [6] of describing the spatial geometry of linkages with respect to a reference frame by matrix multiplications can be used to determine the spatial relationship between the cutting tool and the workpiece, which is the controlling factor of the workpiece accuracy.

As shown in the previous section, any element of the machine tool can be represented by a homogeneous transformation matrix, which describes the relative position and orientation of a coordinate frame embedded in this element with respect to another coordinate frame embedded in another element of the machine. Thus, by multiplying the homogeneous transformation matrices corresponding to a series of elements such as carriage, cross slide, cutting tool, workpiece and spindle, one can describe the tip of the cutting tool and the point on the workpiece which has to be in contact with the cutting tool, with respect to a conveniently chosen 
fixed reference frame. Ideally, the resultant homogeneous transformation matrices, $T_{T O O L}$ for the cutting tool and $T_{W O R K}$ for the point on the workpiece, should be identical. However, due to the errors involved in the machining operation such as the geometric and thermal errors of the machine tool, fixturing and tool wear, two matrices describe the two separate coordinate frames. This causes an erroneous cutting operation and degrades the accuracy of the workpiece being machined. This error, $E$, is represented by the following equation:

$$
T_{\text {WORK }}=T_{\text {TOOL }} E
$$

Thus, the equation

$$
E=T_{\text {TOOL }}^{-1} T_{\text {WORK }}
$$

gives the resultant error matrix, which is in the form of Equation 2.1. Thus the position vector $p_{e}$ of the resultant error matrix $E$ needs to be compensated by the error compensation system. An application of this approach to a two-axis turning machine will now be given.

The schematic of this type of machine tool is shown in Figure 2.2. The structure of the machine consists of a spindle, a carriage, a cross slide, and a tool turret. Note that motion of the toolturret joint is restricted when there is a cutting action. Finally, there is also a cutting tool, which is rigidly connected to the tool turret, and a workpiece, which is rigidly connected to the spindle. Figure 2.2 also shows the locations of the coordinate frames, which are assigned to each element of the machine-tool-workpiece system. In this figure, the reference frame is chosen to coincide with the spindle frame at the spindle nose; when the machine is cold, ${ }^{0} T_{z}$ is the carriage frame with respect to the reference frame, ${ }^{z} T_{x}$ is the cross slide frame with respect to the carriage, ${ }^{x} T_{t}$ is the tool turret frame with respect to the cross slide, ${ }^{t} T_{c}$ is the cutting tool frame with respect to the tool turret, ${ }^{0} T_{s}$ is the spindle frame with respect to the reference frame, and ${ }^{s} T_{w}$ is the workpiece frame with respect to the spindle. The ideal homogeneous transformation matrices for these elements are given in the following: 


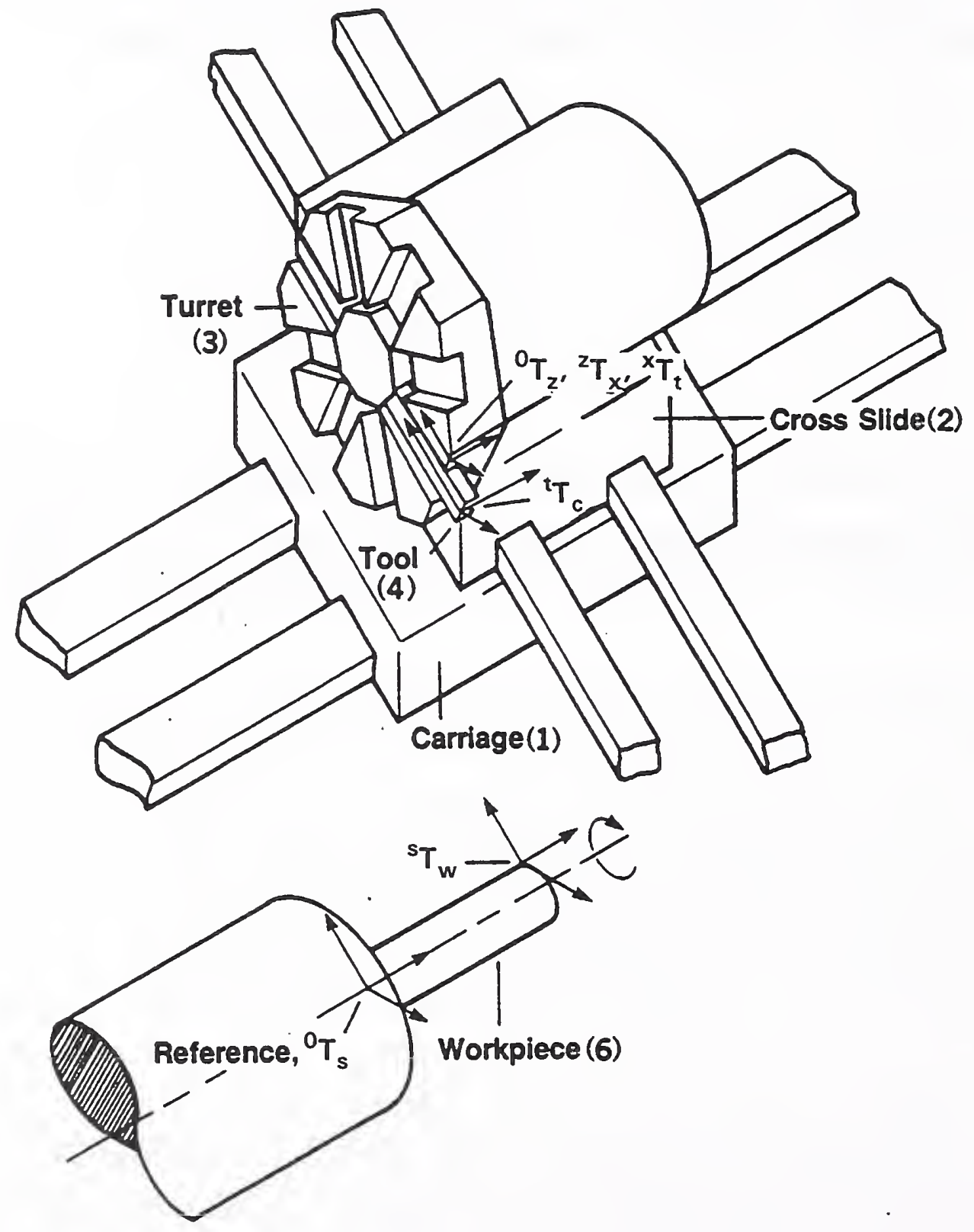

Figure 2.2 Coordinate frame assignments on a two-axis turning center 
For the carriage: the only degree of freedom is in the z-direction.

$$
{ }^{0} T_{z}=\left[\begin{array}{cccc}
1 & 0 & 0 & X_{1} \\
0 & 1 & 0 & 0 \\
0 & 0 & 1 & z \\
0 & 0 & 0 & 1
\end{array}\right]
$$

where,

$z$ is the motion along the $z$-axis (variable),

$X_{1}$ is a constant offset.

For the cross slide: the only degree of freedom is in the x-direction.

$$
{ }^{z} T_{x}=\left[\begin{array}{llll}
1 & 0 & 0 & x \\
0 & 1 & 0 & 0 \\
0 & 0 & 1 & 0 \\
0 & 0 & 0 & 1
\end{array}\right]
$$

where,

$x$ is the motion along the $\mathrm{x}$-axis.

For the tool turret: since all the turret motions are restricted during cutting,

$$
{ }^{x} T_{t}=\left[\begin{array}{llll}
1 & 0 & 0 & 0 \\
0 & 1 & 0 & 0 \\
0 & 0 & 1 & 0 \\
0 & 0 & 0 & 1
\end{array}\right]
$$

For the cutting tool: since it is rigidly attached to the turret,

$$
{ }^{t} T_{c}=\left[\begin{array}{cccc}
1 & 0 & 0 & X_{4} \\
0 & 1 & 0 & 0 \\
0 & 0 & 1 & Z_{4} \\
0 & 0 & 0 & 1
\end{array}\right]
$$


where, $X_{4}$ and $Z_{4}$ are tool dimensions (constants).

For the spindle: although there is a rotational motion about the $\mathrm{z}$ axis during cutting, since there is no significance to the angular position about $\mathrm{z}$ axis, this motion is assumed to be zero.

$$
{ }^{0} T_{s}=\left[\begin{array}{llll}
1 & 0 & 0 & 0 \\
0 & 1 & 0 & 0 \\
0 & 0 & 1 & 0 \\
0 & 0 & 0 & 1
\end{array}\right]
$$

For the workpiece: since it is rigidly attached to the spindle,

$$
{ }^{s} T_{w}=\left[\begin{array}{cccc}
1 & 0 & 0 & x(w) \\
0 & 1 & 0 & 0 \\
0 & 0 & 1 & z(w) \\
0 & 0 & 0 & 1
\end{array}\right]
$$

where, $x(w)$ and $z(w)$ are the coordinates of the ideal cutting point on the workpiece.

On the other hand, the homogeneous transformation matrices representing the actual position and orientation of each element can be obtained, using the error matrices derived in the previous section. This is shown in the following:

For the carriage :

$$
\begin{aligned}
& { }^{0} T_{z}=\left[\begin{array}{cccc}
1 & 0 & 0 & X_{1} \\
0 & 1 & 0 & 0 \\
0 & 0 & 1 & z \\
0 & 0 & 0 & 1
\end{array}\right] \quad\left[\begin{array}{cccc}
1 & -\varepsilon_{z}(z) & \varepsilon_{y}(z) & \delta_{x}(z) \\
\varepsilon_{z}(z) & 1 & -\varepsilon_{x}(z) & \delta_{y}(z) \\
-\varepsilon_{y}(z) & \varepsilon_{x}(z) & 1 & \delta_{x}(z) \\
0 & 0 & 0 & 1
\end{array}\right] \\
& { }^{0} T_{z}=\left[\begin{array}{cccc}
1 & -\varepsilon_{z}(z) & \varepsilon_{y}(z) & \delta_{x}(z)+X_{1} \\
\varepsilon_{z}(z) & 1 & -\varepsilon_{x}(z) & \delta_{y}(z) \\
-\varepsilon_{y}(z) & \varepsilon_{x}(z) & 1 & \delta_{z}(z)+z \\
0 & 0 & 0 & 1
\end{array}\right]
\end{aligned}
$$


where,

$\epsilon_{z}(z)$ is the roll error of carriage (z) motion,

$\epsilon_{x}(z)$ is the pitch error of carriage $(z)$ motion,

$\epsilon_{y}(z)$ is the yaw error of cariage $(z)$ motion,

$\delta_{y}(z)$ is the y straightness of carriage $(z)$ motion,

$\delta_{z}(z)$ is the displacement error of carriage $(z)$ motion,

$\delta_{x}(z)=\delta_{x}^{\prime}(z)+\alpha_{p} \Delta z$

where

$\delta_{x}^{\prime}(z)$ is the $x$ straightness of carriage $(z)$ motion,

$\alpha_{p}$ is parallellism error between $z$ motion and the axis average line of the spindle, and $\Delta z$ is the incremental $z$ motion.

For the cross slide:

$$
\begin{aligned}
& { }^{z} T_{x}=\left[\begin{array}{llll}
1 & 0 & 0 & x \\
0 & 1 & 0 & 0 \\
0 & 0 & 1 & 0 \\
0 & 0 & 0 & 1
\end{array}\right] \quad\left[\begin{array}{cccc}
1 & -\varepsilon_{z}(x) & \varepsilon_{y}(x) & \delta_{x}(x) \\
\varepsilon_{z}(x) & 1 & -\varepsilon_{x}(x) & \delta_{y}(x) \\
-\varepsilon_{y}(x) & \varepsilon_{x}(x) & 1 & \delta_{z}(x) \\
0 & 0 & 0 & 1
\end{array}\right] \\
& { }^{z} T_{x}=\left[\begin{array}{cccc}
1 & -\varepsilon_{z}(x) & \varepsilon_{y}(x) & \delta_{x}(x)+x \\
\varepsilon_{z}(x) & 1 & -\varepsilon_{x}(x) & \delta_{y}(x) \\
-\varepsilon_{y}(x) & \varepsilon_{x}(x) & 1 & \delta_{z}(x) \\
0 & 0 & 0 & 1
\end{array}\right]
\end{aligned}
$$

where,

$\epsilon_{x}(x)$ is the roll error of cross slide $(x)$ motion,

$\epsilon_{z}(x)$ is the pitch error of cross slide $(x)$ motion,

$\epsilon_{y}(x)$ is the yaw error of cross slide $(x)$ motion,

$\delta_{y}(x)$ is the $y$ straightness of cross slide $(x)$ motion,

$\delta_{x}(x)$ is the displacement error of cross slide $(x)$ motion,

$\delta_{z}(x)=\delta_{z}^{\prime}(x)+\alpha_{o} \Delta x$ 
where,

$\delta_{z}^{\prime}(x)$ is the $z$ straightness of cross slide $(x)$ motion,

$\alpha_{o}$ is the orthogonality error between $x$ motion and the axis average line of the spindle, and

$\Delta x$ is the incremental $x$ motion.

For tool turret:

$$
\begin{aligned}
{ }^{x} T_{t} & =\left[\begin{array}{llll}
1 & 0 & 0 & 0 \\
0 & 1 & 0 & 0 \\
0 & 0 & 1 & 0 \\
0 & 0 & 0 & 1
\end{array}\right] \quad\left[\begin{array}{cccc}
1 & -\varepsilon_{z}(t) & \varepsilon_{y}(t) & \delta_{x}(t) \\
\varepsilon_{z}(t) & 1 & -\varepsilon_{x}(t) & \delta_{y}(t) \\
-\varepsilon_{y}(t) & \varepsilon_{x}(t) & 1 & \delta_{z}(t) \\
0 & 0 & 0 & 1
\end{array}\right] \\
{ }^{x} T_{t} & =\left[\begin{array}{cccc}
1 & -\varepsilon_{z}(t) & \varepsilon_{y}(t) & \delta_{x}(t) \\
\varepsilon_{z}(t) & 1 & -\varepsilon_{x}(t) & \delta_{y}(t) \\
-\varepsilon_{y}(t) & \varepsilon_{x}(t) & 1 & \delta_{z}(t) \\
0 & 0 & 0 & 1
\end{array}\right]
\end{aligned}
$$

where,

$$
\begin{aligned}
& \delta_{x}(t), \delta_{y}(t) \text { and } \delta_{z}(t) \text { are translational error motions of the turret, and } \\
& \epsilon_{x}(t), \epsilon_{y}(t) \text {, and } \epsilon_{z}(t) \text { are rotational error motions of the turret. }
\end{aligned}
$$

For the cutting tool: since the coordinate frame is assigned at the tip of the tool, and rotational errors have no effect, 


$$
\begin{aligned}
{ }^{t} T_{c} & =\left[\begin{array}{cccc}
1 & 0 & 0 & X_{c} \\
0 & 1 & 0 & 0 \\
0 & 0 & 1 & Z_{c} \\
0 & 0 & 0 & 1
\end{array}\right]\left[\begin{array}{cccc}
1 & 0 & 0 & \delta_{x}(c) \\
0 & 1 & 0 & \delta_{y}(c) \\
0 & 0 & 1 & \delta_{z}(c) \\
0 & 0 & 0 & 1
\end{array}\right] \\
{ }^{t} T_{c} & =\left[\begin{array}{cccc}
1 & 0 & 0 & X_{4}+\delta_{x}(c) \\
0 & 1 & 0 & \delta_{y}(c) \\
0 & 0 & 1 & Z_{4}+\delta_{z}(c) \\
0 & 0 & 0 & 1
\end{array}\right]
\end{aligned}
$$

where,

$X_{4}$ and $Z_{4}$ are the original tool dimensions in the $x$ and $z$ directions respectively, $\delta_{x}(c), \delta_{y}(c)$, and $\delta_{z}(c)$ are the changes in the tool length in the $x, y$, and $Z$ directions respectively.

For the spindle, due to unrestricted rotational motion about the z-axis, assume $\epsilon_{z}(s)=0$,

$$
\begin{aligned}
{ }^{0} T_{s} & =\left[\begin{array}{llll}
1 & 0 & 0 & 0 \\
0 & 1 & 0 & 0 \\
0 & 0 & 1 & 0 \\
0 & 0 & 0 & 1
\end{array}\right]\left[\begin{array}{cccc}
1 & 0 & \varepsilon_{y}(s) & \delta_{x}(s) \\
0 & 1 & -\varepsilon_{x}(s) & \delta_{y}(s) \\
-\varepsilon_{y}(s) & \varepsilon_{x}(s) & 1 & \delta_{z}(s) \\
0 & 0 & 0 & 1
\end{array}\right] \\
{ }^{0} T_{s} & =\left[\begin{array}{cccc}
1 & 0 & \varepsilon_{y}(s) & \delta_{x}(s) \\
0 & 1 & -\varepsilon_{x}(s) & \delta_{y}(s) \\
-\varepsilon_{y}(s) & \varepsilon_{x}(s) & 1 & \delta_{z}(s) \\
0 & 0 & 0 & 1
\end{array}\right]
\end{aligned}
$$

where,

$\delta_{x}(s)$ is the radial motion of spindle in sensitive direction,

$\delta_{y}(s)$ is the radial motion of spindle in nonsensitive direction,

$\delta_{z}(s)$ is the axial motion of the spindle, 
$\epsilon_{x}(s)$ is the tilt of the spindle in nonsensitive direction,

$\epsilon_{y}(s)$ is the tilt of the spindle in sensitive direction.

Note that only thermal drift components of these error motions are considered. Since existing machine tool controllers do not have sufficient bandwidth, the dynamic spindle errors are not included in the compensation model.

For the workpiece, assuming no rotational errors,

$$
\begin{aligned}
& { }^{s} T_{w}=\left[\begin{array}{cccc}
1 & 0 & 0 & x(w) \\
0 & 1 & 0 & 0 \\
0 & 0 & 1 & z(w) \\
0 & 0 & 0 & 1
\end{array}\right]\left[\begin{array}{cccc}
1 & 0 & 0 & \delta_{x}(w) \\
0 & 1 & 0 & \delta_{y}(w) \\
0 & 0 & 1 & \delta_{z}(w) \\
0 & 0 & 0 & 1
\end{array}\right] \\
& { }^{s} T_{w}=\left[\begin{array}{cccc}
1 & 0 & 0 & x(w)+\delta_{x}(w) \\
0 & 1 & 0 & \delta_{y}(w) \\
0 & 0 & 1 & z(w)+\delta_{z}(w) \\
0 & 0 & 0 & 1
\end{array}\right]
\end{aligned}
$$

where,

$x(w)$ and $z(w)$ are the coordinates of the point on the workpiece which ideally interacts with the cutting tool to obtain desired workpiece geometry, $\delta_{x}(w), \delta_{y}(w)$, and $\delta_{z}(w)$ are the changes of the coordinates of this point in the $x, y, z$ directions respectively.

Using these transformation matrices, the cutting tool with respect to the reference frame is represented by the following matrix multiplication:

$$
T_{\text {TOOL }}={ }^{o} T_{z}{ }^{z} T_{x}{ }^{x} T_{t}{ }^{t} T_{c}
$$

Similarly, the ideal workpiece-cutting tool interface point on the workpiece with respect to the reference frame is given by: 


$$
T_{\text {WORK }}={ }^{O} T_{s}{ }^{s} T_{w}
$$

Finally, the error matrix $E$ is calculated using Equation 2.9. The matrix $E$ is also a homogeneous transformation matrix in the form of Equation 2.1, which consists of position and orientation vectors $o_{1}, o_{2}, o_{3}$, and $p$. Carrying out the matrix multiplications in Equations 2.22, 2.23, and 2.9, the three components of the position vector $p_{e}$ of the error matrix $E$ can be calculated as,

$$
\begin{gathered}
p_{E x}=x(w)+\delta_{x}(w)+\varepsilon_{y}(s) * z(w)+\delta_{x}(s)-X_{4}-\delta_{x}(c) \\
-\left[\varepsilon_{y}(z)+\varepsilon_{y}(x)+\varepsilon_{y}(t)\right] * Z_{4}-\delta_{x}(t)-x-X_{1}-\delta_{x}(s)-\delta_{x}(z) \\
p_{E y}=\delta_{y}(w)-\varepsilon_{x}(s) * z(w)+\delta_{y}(s)-\left[\varepsilon_{z}(z)+\varepsilon_{z}(x)+\varepsilon_{z}(t)\right] * X_{4}-\delta_{y}(c) \\
+\left[\varepsilon_{x}(z)+\varepsilon_{x}(x)+\varepsilon_{x}(t)\right] * Z_{4}-\delta_{y}(t)-\varepsilon_{z}(z) * x-\delta_{y}(x)-\delta_{y}(z) \\
p_{E y}=-\varepsilon_{y}(s) * x(w)+z(w)+\delta_{z}(w)+\delta_{z}(s)+\left[\varepsilon_{y}(z)+\varepsilon_{y}(x)+\varepsilon_{y}(t)\right] * X_{4} \\
-Z_{4}-\delta_{z}(c)-\delta_{z}(t)+\varepsilon_{y}(z) * x-\delta_{z}(x)-\delta_{z}(z)-z
\end{gathered}
$$

In Equations 2.24, 2.25 and 2.26:

$x+X_{1}$ and $z$ are the nominal machine positions,

$x(w)$ and $z(w)$ are obtained from workpiece geometry, (desired workpiece dimensions)

$\delta_{x}(w)$ and $\delta_{z}(w)$ are functions of thermal and static load deformations, on the workpiece $\delta_{x}(s), \delta_{z}(s)$, and $\epsilon_{y}(s)$ are spindle thermal drift characteristics,

$\delta_{x}(c), \delta_{z}(c)$ are functions of thermal and load deformations and wear, of the cutting tool $\delta_{x}(t), \delta_{z}(t)$, and $\epsilon_{y}(t)$ are functions of angular position of the turret, $\delta_{x}(x), \delta_{z}(x), \epsilon_{y}(x), \delta_{x}(z), \delta_{z}(z)$, and $\epsilon_{y}(z)$ are functions of machine-tool geometry and thermal characteristics.

If angular orientation of the tool can be modified by the rotational axes of the machine, the orientation vectosis $o_{1}, o_{2}$, and $o_{3}$ can be calculated similarly. Figure 2.3 illustrates the resultant 
error components at the tip of the cutting tool.

The model, which has been applied to a two-axis turning machine, above can be modified for other types of machine tools, such as multiaxes machining centers. The major difference would be additional matrices corresponding to the additional elements of the machine structure, in the Equations 2.22 and 2.23. For example, for a $31 / 2$-axis fixed-column vertical machining center, shown in fig. 2.4:

$$
T_{\text {TOOL }}={ }^{o} T_{w}{ }^{w} T_{z}{ }^{z} T_{s}{ }^{s} T_{c}
$$

where

${ }^{\circ} T_{w}$ is the transformation matrix for $w$-slide

${ }^{w} T_{z}$ is the transformation matrix for $z$-slide

${ }^{z} T_{s}$ is the transformation matrix for spindle

${ }^{s} T_{c}$ is the transformation matrix for cutting tool

$$
T_{\text {WORK }}={ }^{o} T_{y}{ }^{y} T_{x}{ }^{x} T_{p}
$$

where

${ }^{\circ} T_{y}$ is the transformation matrix for $y$-slide

${ }^{y} T_{x}$ is the transformation matrix for $x$-slide

${ }^{x} T_{p}$ is the transformation matrix for workpiece.

Using Equation 2.9, the error vector can be solved for a given tool position in space. An important criteria in the selection of the locations of the coordinate frames is that the relative errors should be easily defined and measured or predicted. Once the model is generated, the next step is to measure and/or predict the individual error components appearing in the resultant error equations, the procedure of which is described in the next section. 


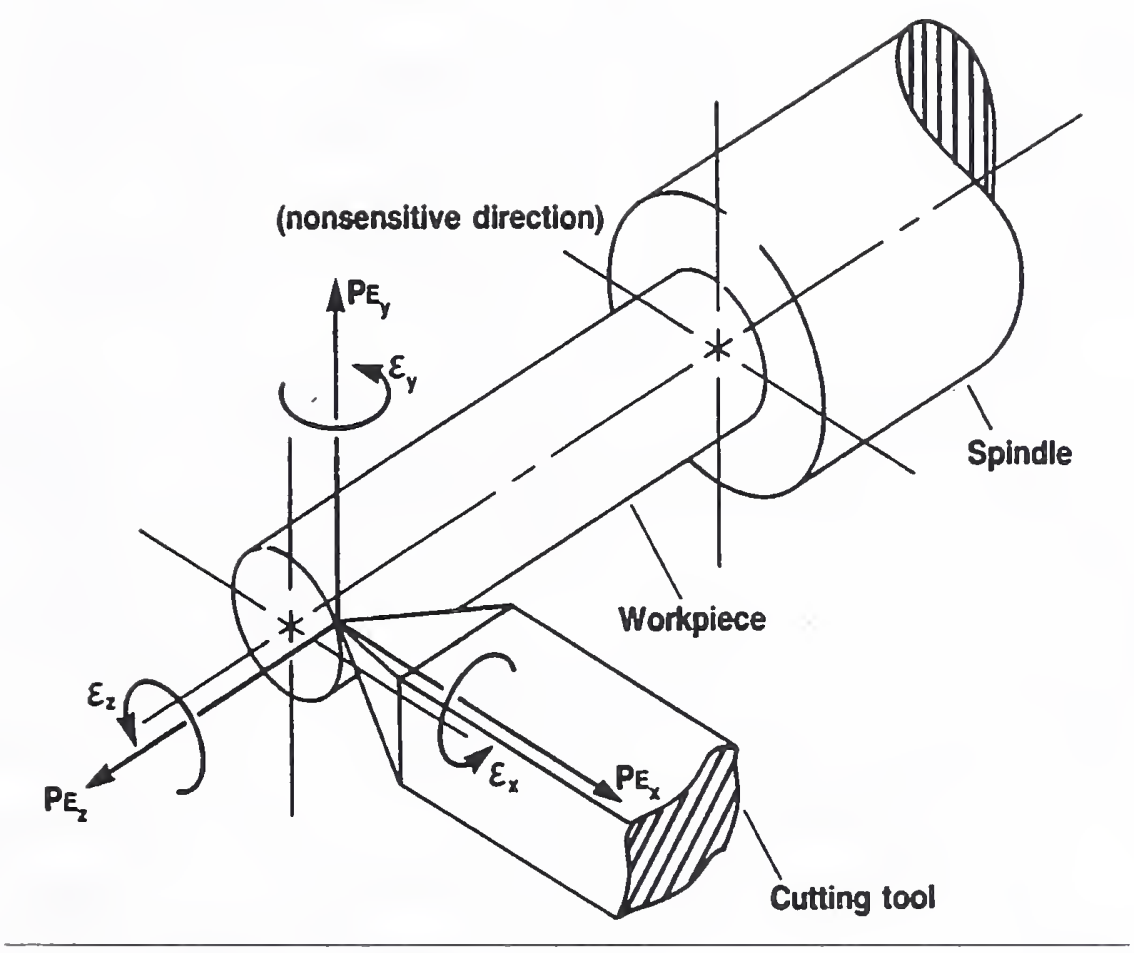

Figure 2.3 The resultant error components at the tip of the cutting tool. (For single point turning operation.) 


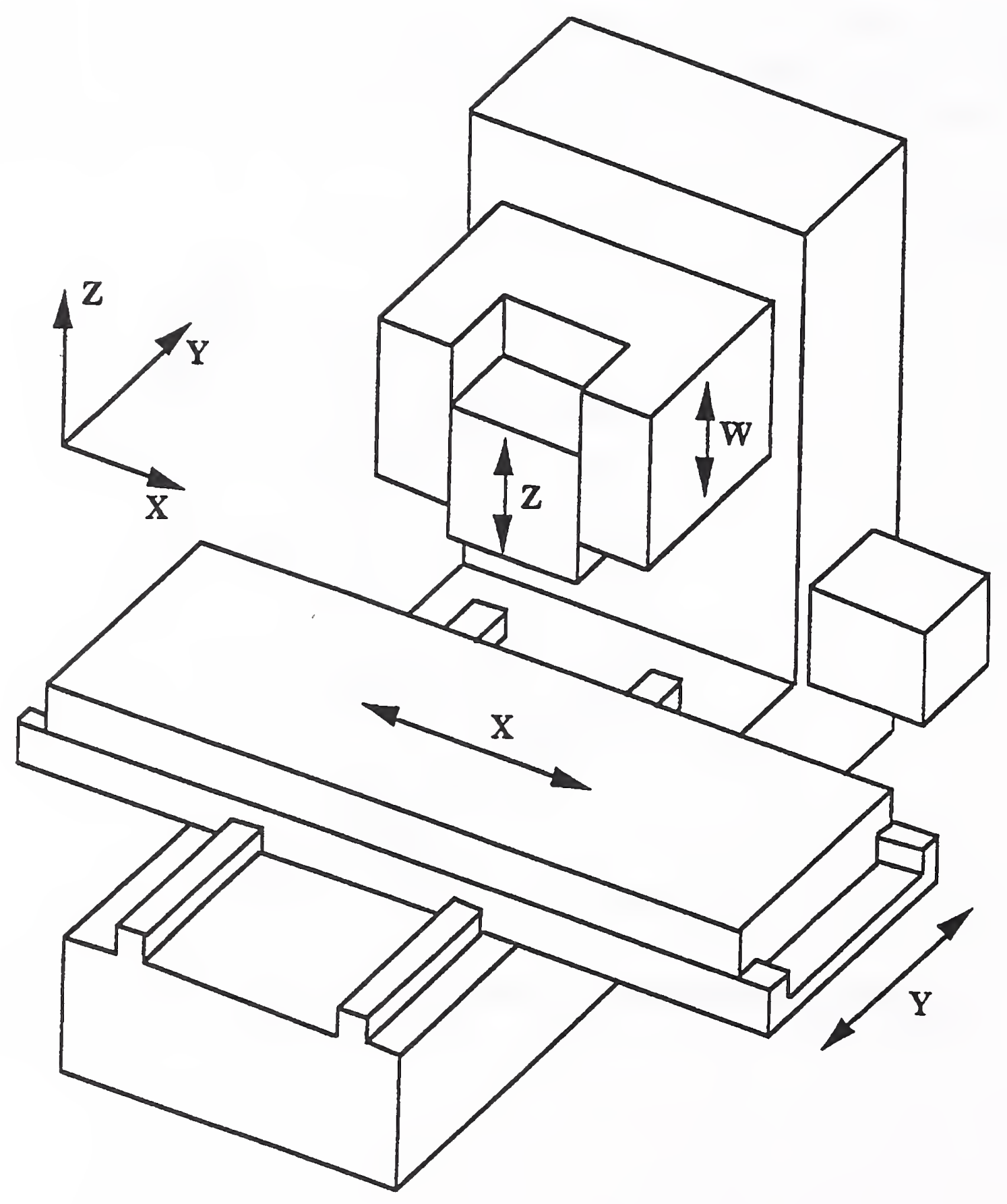

Figure 2.4 Schematic of 3 1/2-axis fixed column vertical machine center 


\section{Machine Characterization}

In the previous section the resultant error at the tip of the cutting tool was calculated as a combination of individual error components corresponding to different structural elements of the machine tool. In order to predict the resultant error at any location and at any time in the machine work space, all of these error components must be predicted. Although these components are geometric error components of the structural elements of the machine tool, their characteristics change as a result of the changing thermal profile of the machine structure. The purpose of the machine characterization is to establish the relationship between the cutting tool and the workpiece under the transient thermal conditions. The techniques and procedures for systematic error measurements of the machine tool will be presented in this section.

\subsection{General Procedure}

Since two independent sets of variables, the nominal position and the thermal state of the machine, affect the geometric errors, measurements must be carried out to find the relationships between these variables over their possible ranges. For each machine axis, the measurement starts when the slide under study is at one end of its travel range. Then the slide is moved toward the other end of its travel range while a reading is taken at every measuring interval. The motion is reversed at the end of the travel, and the slide is sent back to its starting position, again with a reading taken at every measuring interval. With this procedure, it is possible to measure the reversal error of each axis. In selecting the measuring intervals, one has to consider the periodic error components such as those caused by leadscrew misalignment ${ }^{1}$. In order to eliminate the effect of the periodic errors from the measurements, the measuring interval should be selected at even multiples of the leadscrew pitch or the pitch of the linear scale. However, in order to determine the periodic error without thermal contamination of the data, the periodic error measurements should be taken separately over a very-short travel range (one or two times the pitch) with the assumption of uniformity over the whole travel range.

${ }^{1}$ Errors with the leadscrew pitch have been found in a machine with glass scales for position feedback. Such errors can be caused by abbe error. 
Figures 3.1 and 3.2 illustrate the temperature profiles of several locations on a machine structure obtained during a typical warm up cycle. These measurements indicate that approximately 8 to 10 hours are required for a typical machine to reach thermal equilibrium under continuous running conditions in an average machine shop environment. In order to find the thermally-induced changes on the machine errors, these errors must be measured over the operating temperature spectrum of the machine. The error measurements must start when the machine is "cold". After five cycles of measurements, each of which consists of movement over the whole travel range of the axis being measured, the machine is warmed up. Two types of warm up procedures are used. In the first one, the machine is on, but there is no movement. This causes a slow warm-up. In the second one, the warm-up is induced by moving the machine slide back and forth. By using these procedures, the machine is gradually warmed up and the measurements are taken after each warm-up period. During each measurement cycle, in addition to the error movements of the slide under study, the temperatures of the locations around the slide are monitored. The critical locations at which the temperatures are to be monitored are bearing housings at both ends of the leadscrew of the slide, the slideways, the drive motor, the bed on which the slide is moving, the fixture for the position feedback measurement sensor, and the ambient air. Typical locations are shown in fig. 3.3 for a machining center with linear scales. This measurement procedure allows one to investigate the change in the geometric error behavior corresponding to the change in the thermal state of the machine.

The error components in Equations 2.24 through 2.26 can be classified into four groups with respect to characteristic similarities, measurement procedures, and the sensors used. These are:

1. Linear displacement errors

2. Angular errors

3. Straightness - Parallelism - Orthogonality

4. Spindle thermal drift

The measurement techniques and the sensors used for each class are presented in the following sections. 


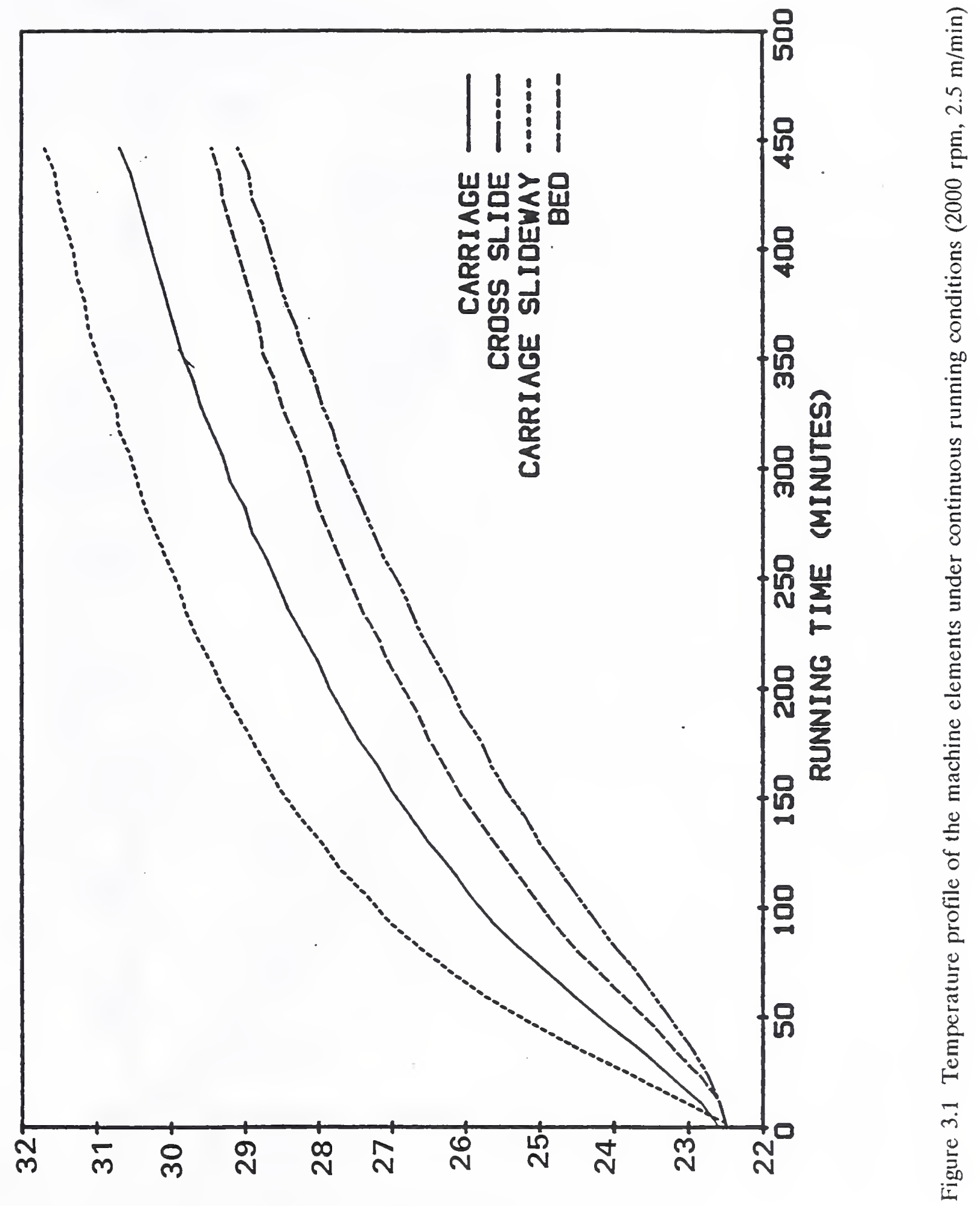

(0 ร $\exists \exists y 9 \exists a) ~ \exists y \cap \perp \forall y \exists d W \exists \perp$ 


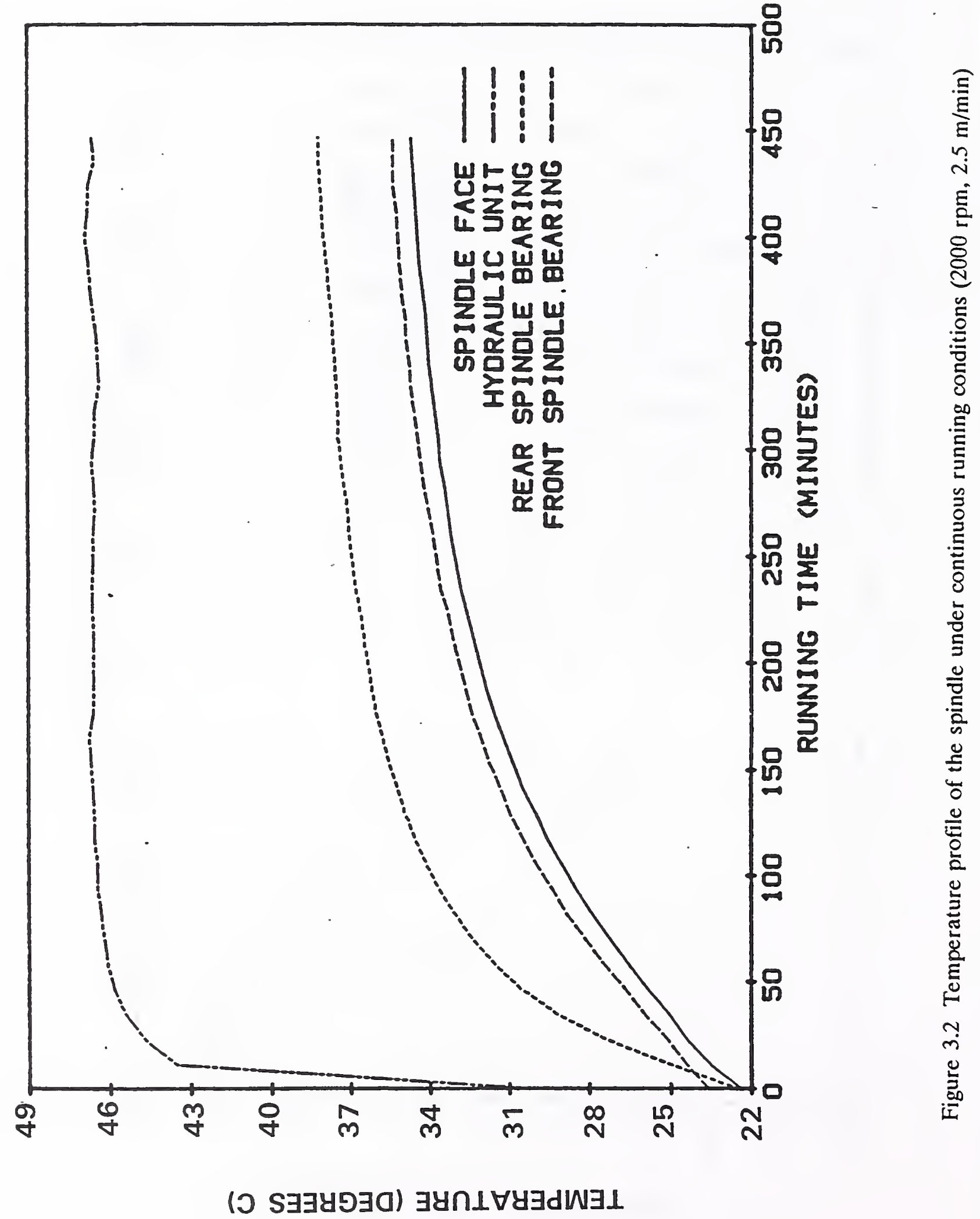




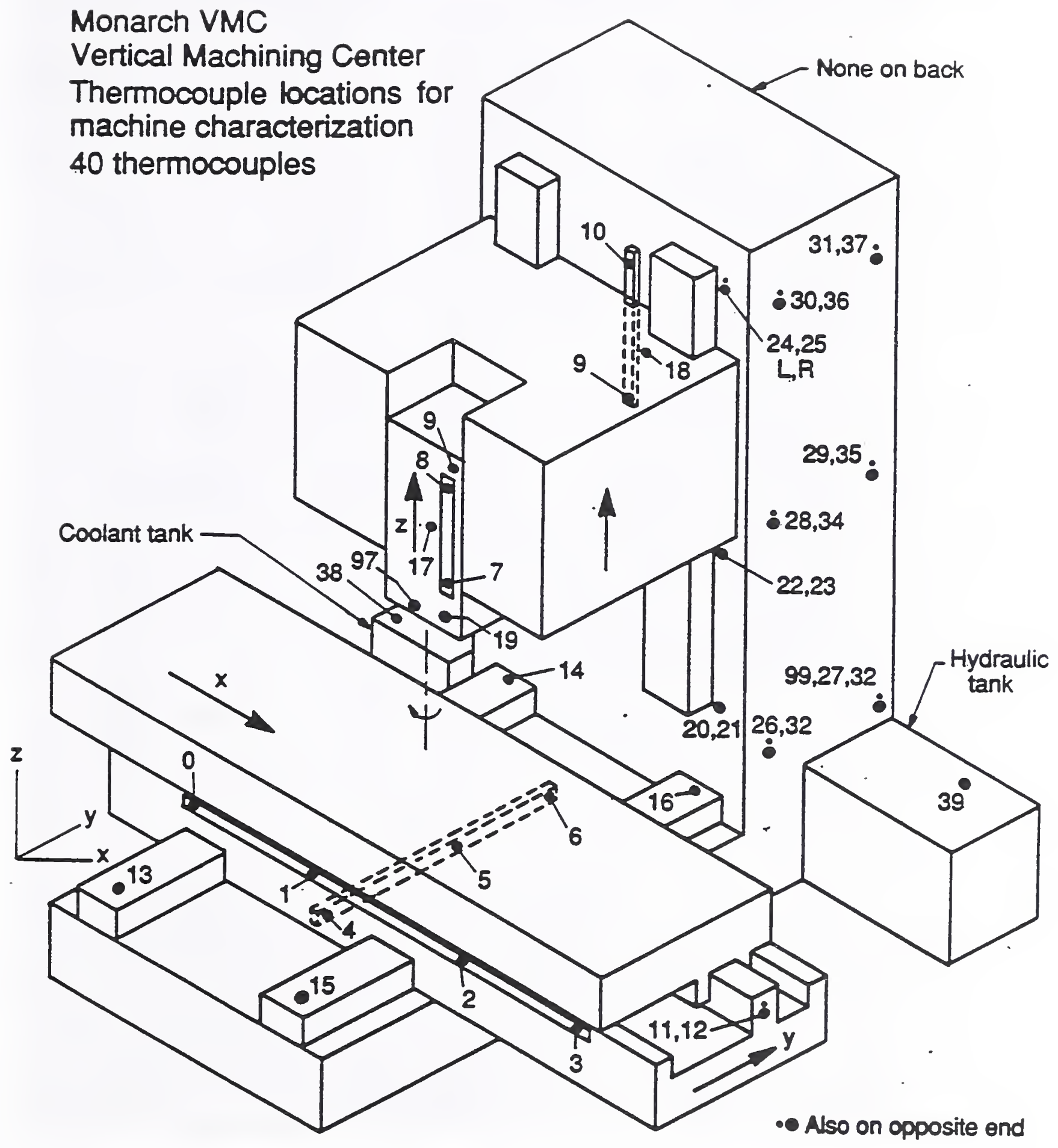

Figure 3.3 Vertical Machining Center Thermocouple locations for machine characterization 40 thermocouples 


\subsection{Linear Displacement Errors}

Linear displacement error is defined as the translational error movement of a machine element along its axis of motion. In general, this type of error is caused by the geometric inaccuracies of the drive mechanism and the position feedback unit. In the case of ballscrew driven slides with rotary position feedback, erroneous pitch of the ballscrew, misalignment between its axis of rotation and its centerline, irregularities in its geometry, and defective coupling between the feedback unit and the ballscrew cause linear displacement errors. With linear position feedback devices, displacement errors can be significantly lower.

The best available device for displacement measurements over the travel ranges of machine slides is the laser interferometer. Such a system is capable of measuring displacements up to $40 \mathrm{~m}$ with $10 \mathrm{~nm}$ resolution and one part in $10^{7}$ accuracy [7]. Figures 3.4 and 3.5 show the setup of the laser interferometer optics for linear displacement measurements along the $\mathrm{x}$ and $\mathrm{z}$ axes of a turning center, respectively. Due to the high- accuracy requirements, the stability of the measuring devices is a very important factor throughout the data acquisition period. Laser interferometer readings are affected mostly by changes in atmospheric pressure and temperature which change the velocity of light. To avoid this error, the pressure and temperature of the ambient air near the laser beam should be monitored during the measurements, and the laser readings compensated accordingly $[8,9]$.

\subsection{Angular Errors}

Angular errors are rotational errors caused by geometric inaccuracies of the slideways and the misalignment in the assemblies of structural elements of the machine tool. The three rotational errors, which are around the three orthogonal axes of the machine slide, are defined as roll, pitch and yaw. Yaw error is the rotational error of the slide around the axis perpendicular to the plane in which axis of motion lies. Roll error is the rotational error of the slide around the axis of motion, and pitch error is the rotational error of the slide around the third othogonal axis of the slide. Although the contributions of all three rotational errors to the resultant error are significant in three or more axes machining centers, in turning centers, the contributions of roll and pitch errors are in a nonsensitive direction, which is the direction perpendicular to the plane in which the two machine slides are moving. Therefore, for turning centers, yaw 


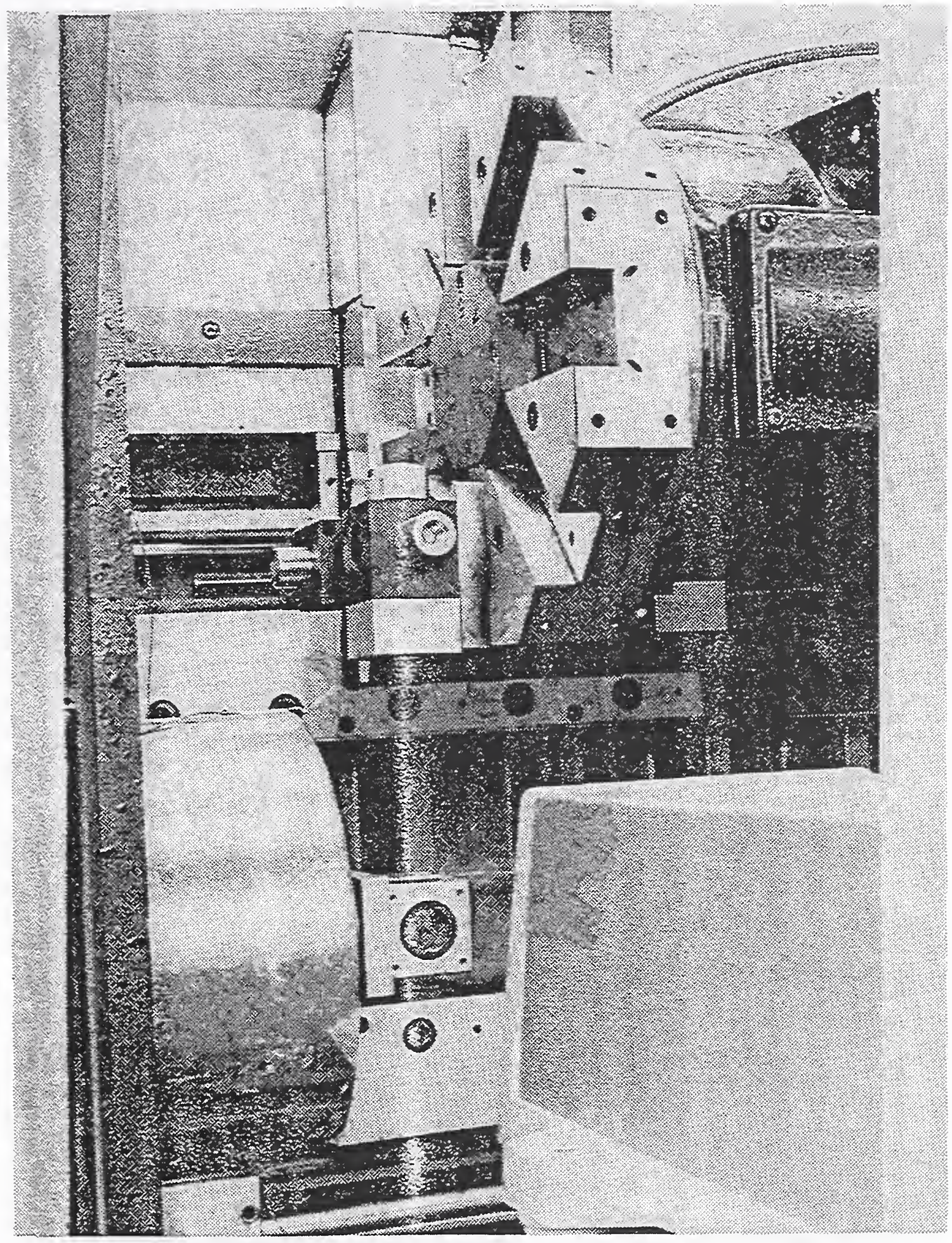

Figure 3.4 The laser interferometer set-up for $\mathrm{x}$ displacement error measurements 


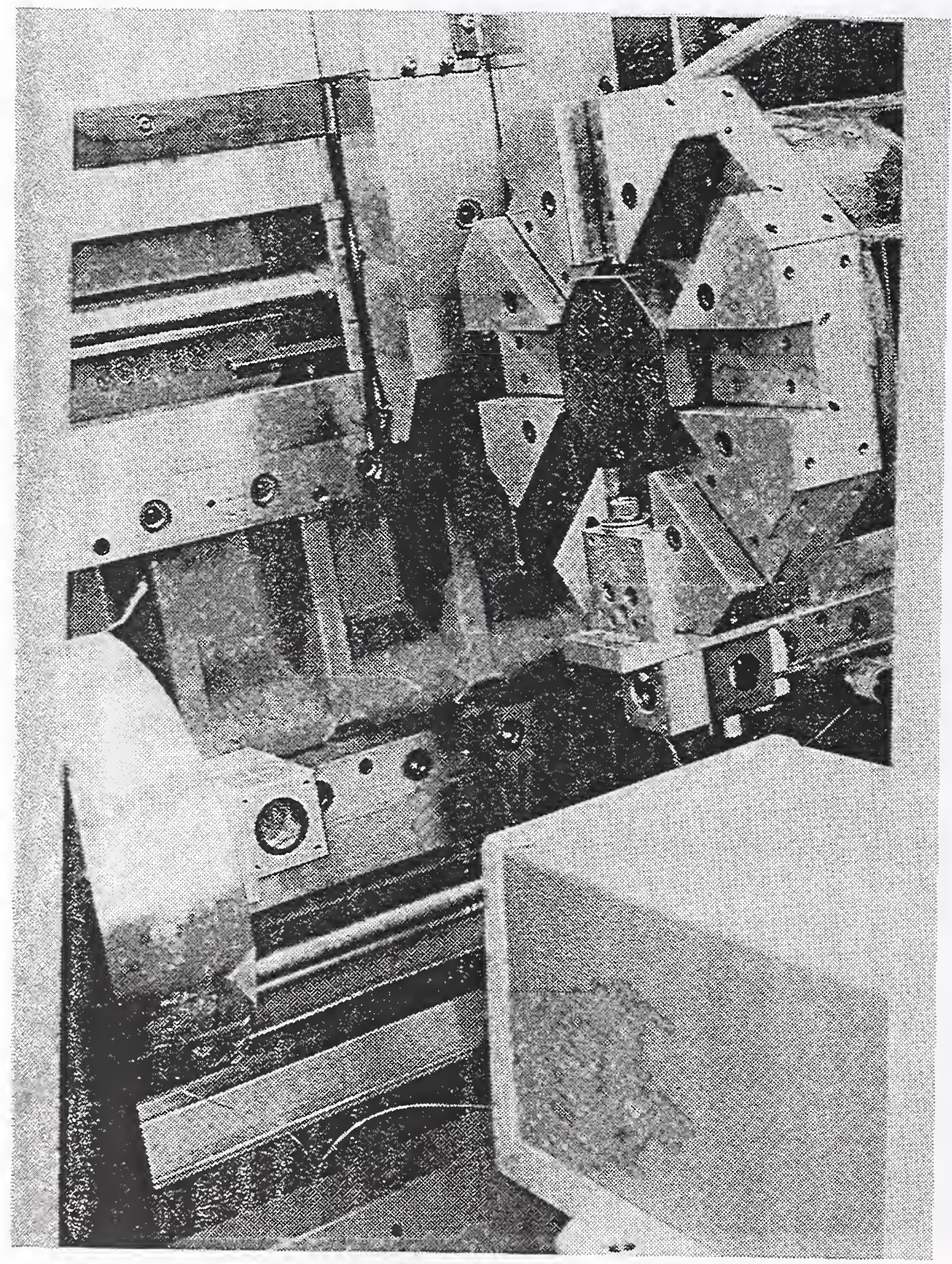

Figure 3.5 The laser interferometer set-up for $z$ displacement error measurements 
error measurements are sufficient for resultant error calculations. Since the laser interferometer is not able to measure the roll error of machine slides, the most common way to measure this rotational error is to use electronic levels. For pitch and yaw error measurements, a laser interferometer is still the best available system. Except for the optical attachments for the laser interferometer, the data acquisition system for the yaw measurements is the same as the one used for the linear displacement error measurements. Since the principle of the measurement is based on the comparison of the path lengths of the laser beam reflected from two retroreflectors, there is no need for velocity of light compensation. The set ups of the laser optics for the cross slide and the carriage yaw error measurements for a turning center are shown in figs. 3.6 and 3.7 respectively.

\subsection{Straightness-Parallelism-Orthogonality}

Straightness is the translational error of the machine element in the two orthogonal directions other than its own axis of motion. The laser interferometer system can be used for straightness measurements, however, the size of the optical attachments required, and the minimum allowable distance between them (the Wollastron prism has to be at least $100 \mathrm{~mm}$ away from the reflector) limits its use, especially in turning centers which have short cross slide travel ranges. Therefore, high-precision proximity probes and straightness artifacts such as precision manufactured test arbors can also be used for this group of measurements. The techniques used for the measurements using a test arbor are explained in the following.

A sample set up for the measurements of $x$ straightness of $z$ motion are shown in Figure 3.8. The principle of this measurement is to measure the change in the gap between the probe, which is attached to the carriage while it is moving along the $\mathrm{z}$ axis, and the test arbor mounted on the spindle. Although a precision ground test arbor is used for this purpose, there are errors involved with this type of measurement. In this case, in addition to the axis straightness error, the probe output includes nonstraightness of the test arbor profile, and the misalignment between the arbor and the spindle. To eliminate the arbor profile error, the reversal technique is used [11]. The best fit slope subtraction on the resultant data eliminates the misalignment errors. 


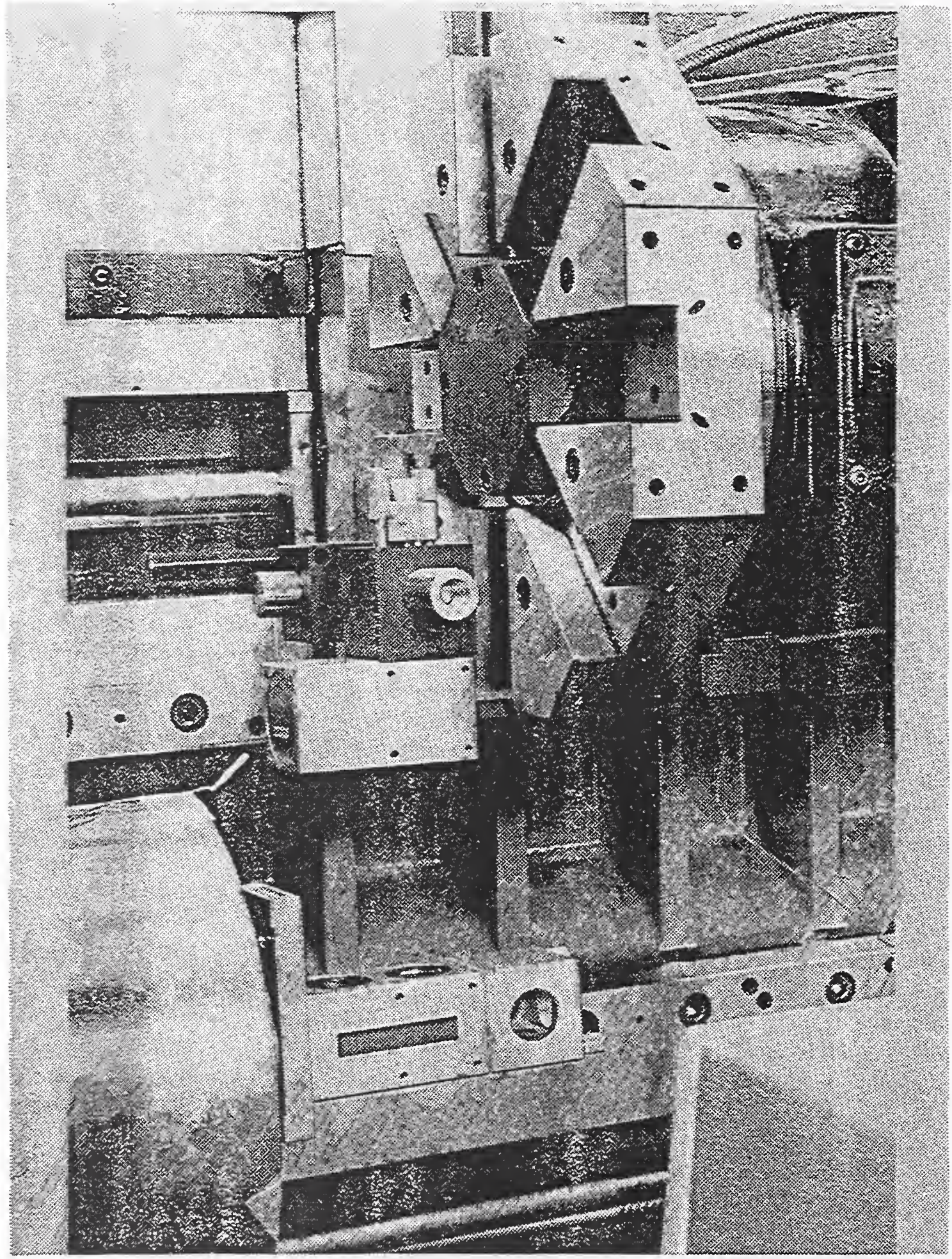

Figure 3.6 The laser interfereometer set-up for $\mathrm{x}$ yaw error measurements 


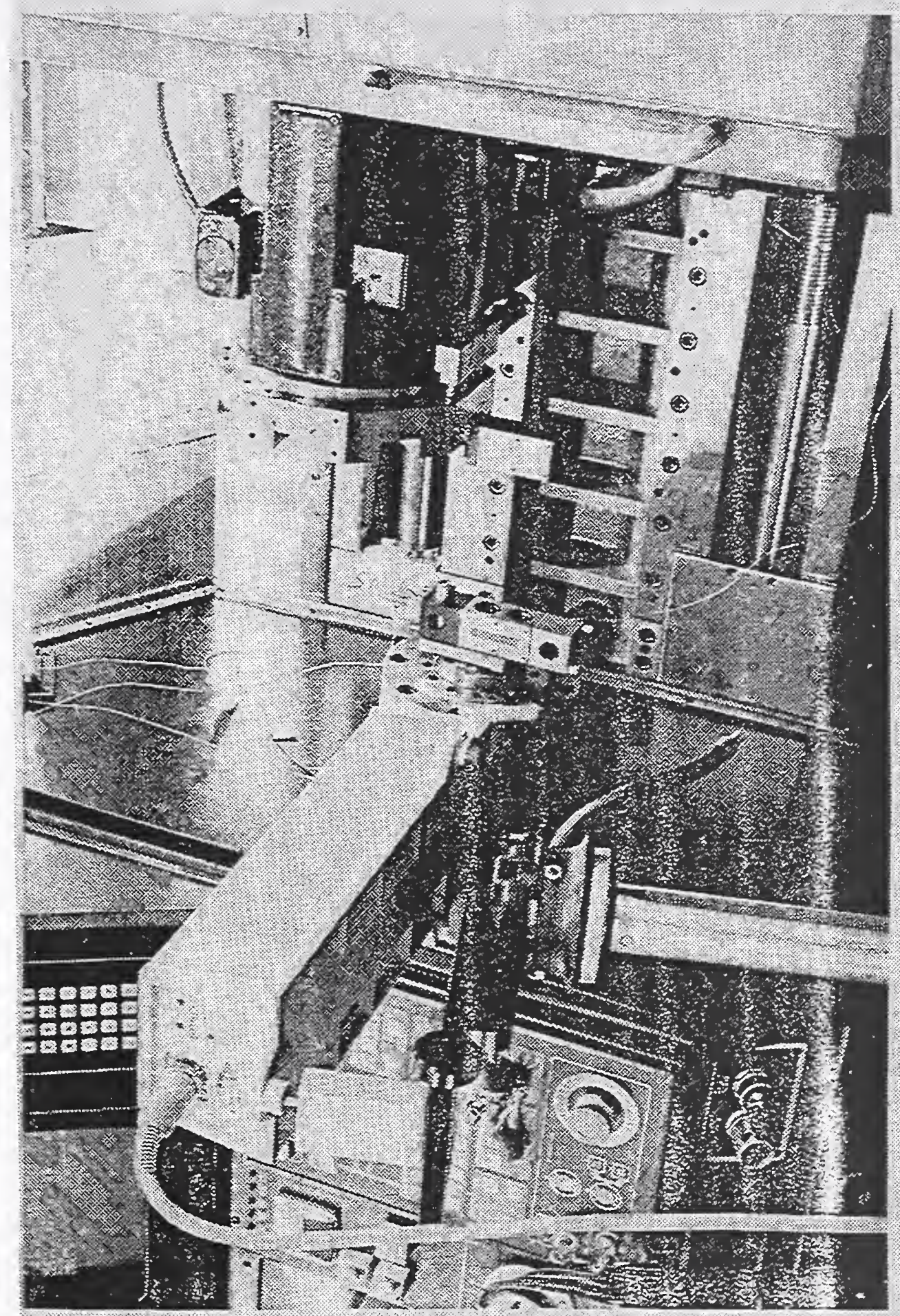

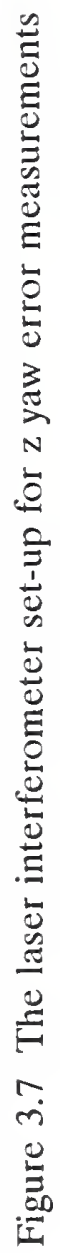



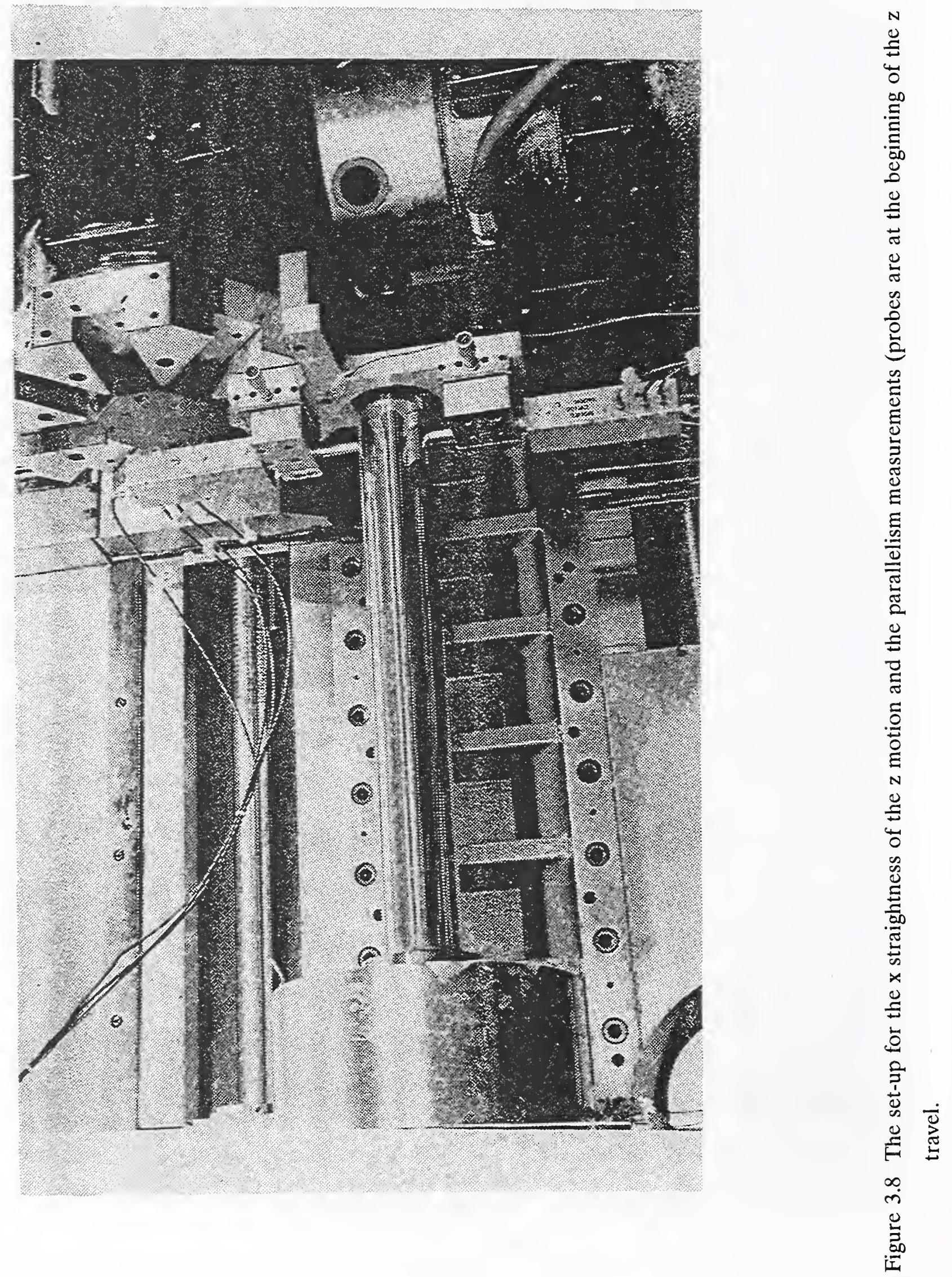
In order to apply the reversal technique, two sets of measurements are required along the $z$ axis. In the first set, the carriage is moved along the $z$ axis taking the probe outputs at every measuring interval. After the first set, the test arbor is rotated 180 degrees with the spindle. In the second set, outputs of the second probe (which is mounted 180 degrees away from the first probe) are recorded. At any point along the $z$ axis, the first measurement is represented by the following equation:

$$
m_{1}(z)=a(z)-s(z)
$$

where,

$m_{1}(z)$ is the output of probe 1 at position $z$,

$a(z)$ is the profile nonstraightness of the test arbor,

$s(z)$ is the straightness of $z$ motion.

Similarly, the second measurement is given as:

$$
m_{2}(z)=a(z)+s(z)
$$

where,

$m_{2}(z)$ is the output of probe 2 at position $z$

From Equations 3.1 and 3.2 the straightness of $z$ motion is found as:

$$
s(z)=\frac{m_{2}-m_{1}}{2}
$$

Figure 3.9 shows the schematic of this technique.

The parallelism between the axis of $\mathrm{z}$ motion and the axis average line of the spindle can be determined, ideally, by taking two probe outputs against the test arbor at any two points along the $\mathrm{z}$ axis, and dividing the difference by the length between these points. But, as mentioned in the previous section, there are several errors involved in such a measurement [12]. Z-axis straightness error, test arbor profile error, and the misalignment between the test arbor and the spindle are the major contributors. In order to eliminate these errors, the following procedure 
(a) First set of measurements $m 1(z)$ with Probe 1:

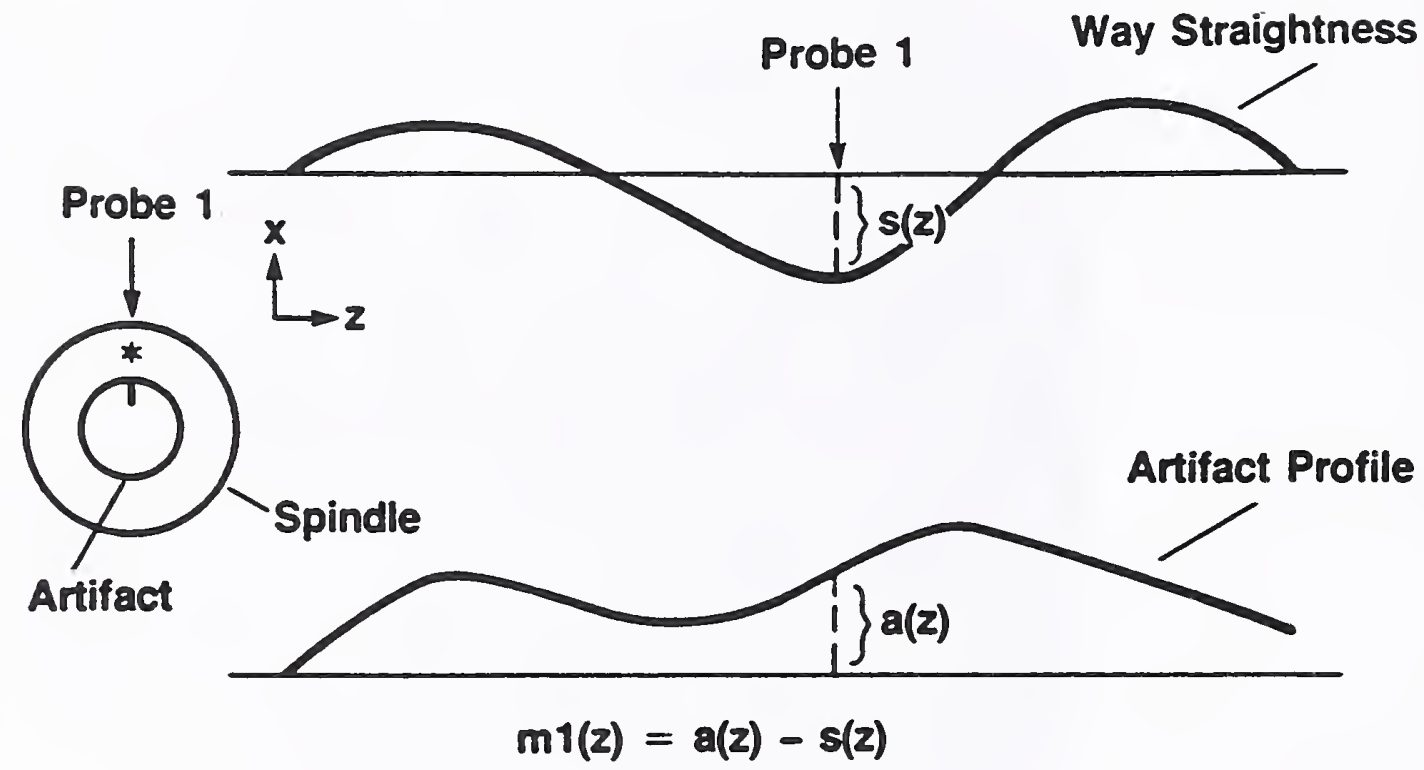

(b) Second set of measurements m2(z) with Probe 2: (Artifact rotated $180^{\circ}$ inside the spindle)

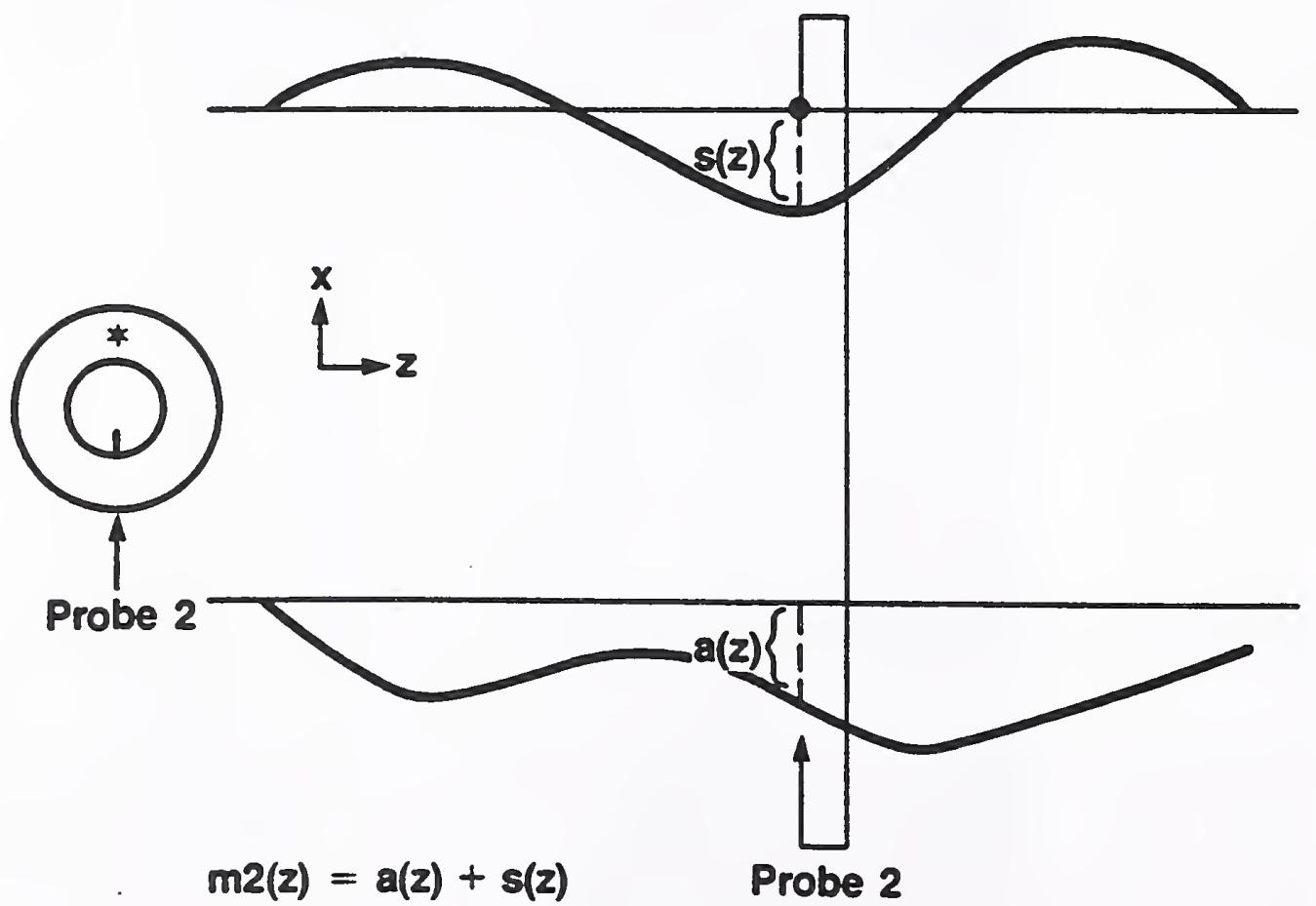

Figure 3.9 The schematic of the $\mathrm{x}$ straightness of the $\mathrm{z}$ motion measurement technique 
with the set-up shown in Figures 3.7 is used. The spindle is rotated at a very-low speed in order to eliminate the spindle error motions, and, using an analog-to-digital (A/D) converter, the capacitance probe outputs at 512 points (for example) in one revolution are recorded for each probe at two locations, $300 \mathrm{~mm}$ apart, along the $\mathrm{z}$ axis. It is known that the misalignment between a perfectly round artifact and a spindle with no error motion creates a limacon as an output of such a measurement [12]. The artifact nonroundness and the spindle error distorts this limacon. In order to eliminate the effects of these errors on the data, a best fit circle out of 512 data points for one revolution of spindle is calculated and used for the analysis. The calculation of best fit circle radius from the data is performed using the following formula [12].

$$
R=\left(\sum r_{i}\right) / n
$$

where,

$r_{i}$ is the probe output at the angular position $i$

$n$ is the number of data points,

$R$ is the best-fit radius.

To eliminate the axis straightness errors, these best fit circles are constructed by the outputs of two probes 180 degrees apart. The parallelism error is then calculated using the following formula:

$$
\alpha_{p}=\left[\left(R_{21}-R_{11}\right)-\left(R_{22}-R_{12}\right)\right] / 2 \Delta z
$$

where,

$\alpha_{\mathrm{p}}$ is the parallelism error

$R_{11}$ is the least square radius from probe 1 at location 1 , $R_{21}$ is the least square radius from probe 1 at location 2 , $R_{12}$ is the least square radius from probe 2 at location 1 , $R_{22}$ is the least square radius from probe 2 at location 2 , $\Delta z$ is the distance between location 1 and location 2 .

The schematic of the probe arrangements is shown in Figure 3.10. 


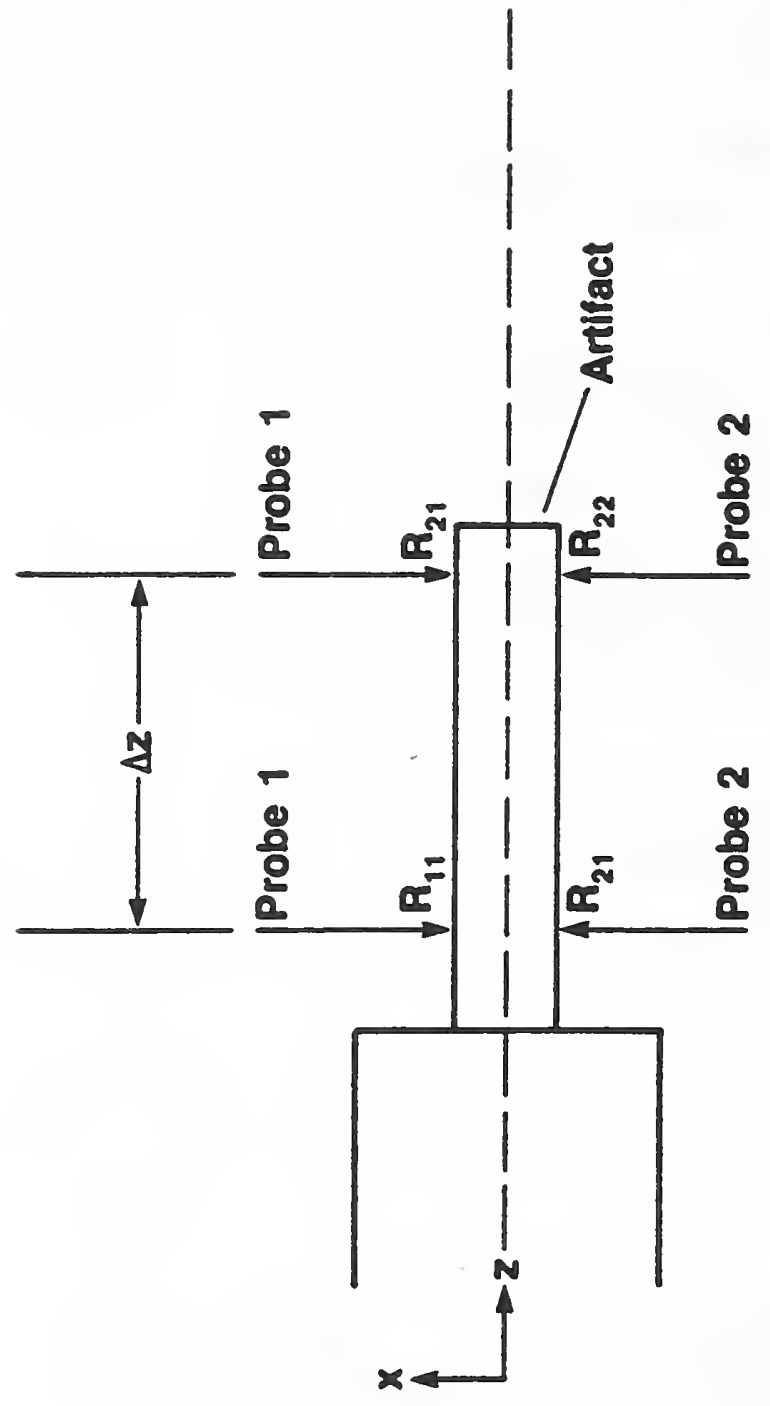

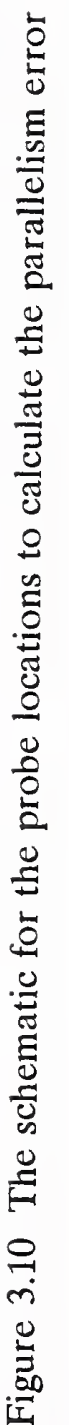


A different type of test artifact is used for the measurements of the $z$ straightness of the $x$ motion and the orthogonality of the $x$ axis to the axis average line of the spindle. The artifact is a disk with a ground and lapped flat face which can be scanned. It is not possible to measure the back side of this artifact while mounted on the spindle. Hence, it is impossible to apply the reversal technique to eliminate artifact profile errors from the straightness measurements. The flat face of this arbor must be calibrated before its use. This can be done on a coordinate measuring machine using the reversal technique, this time to eliminate machine straightness errors. This arbor is mounted on the spindle and a probe is mounted on the turret, as shown in Figure 3.11. In order to eliminate the misalignment and the arbor squareness errors, a new technique can be used. While the cross slide is moving along the face of the test arbor, the probe readings are taken at every measuring interval. Then, the spindle is rotated 180 degrees and the measurement repeated with the same probe. With this procedure, it is possible to find the orthogonality as well as the $\mathrm{z}$ straightness of the $\mathrm{x}$ motion. The following relationships are obtained from the geometry shown in Figure 3.12 (assuming perfect profile).

$$
\begin{aligned}
& m_{1}(x)=-\delta_{z}(x)+e(x) \\
& m_{2}(x)=-\delta_{z}(x)-e(x)
\end{aligned}
$$

where,

$m_{1}(x)$ is the probe output for the first set of measurements,

$m_{2}(x)$ is the probe output after the spindle rotated 180 degrees,

$\delta_{z}(x)$ is the $z$ straightness of $x$ motion,

$e(x)$ is the combination of arbor squareness and misalignment errors.

From equations 3.6 and 3.7 the straightness is calculated:

$$
\delta_{z}(x)=-\left[m_{1}(x)+m_{2}(x)\right] / 2
$$




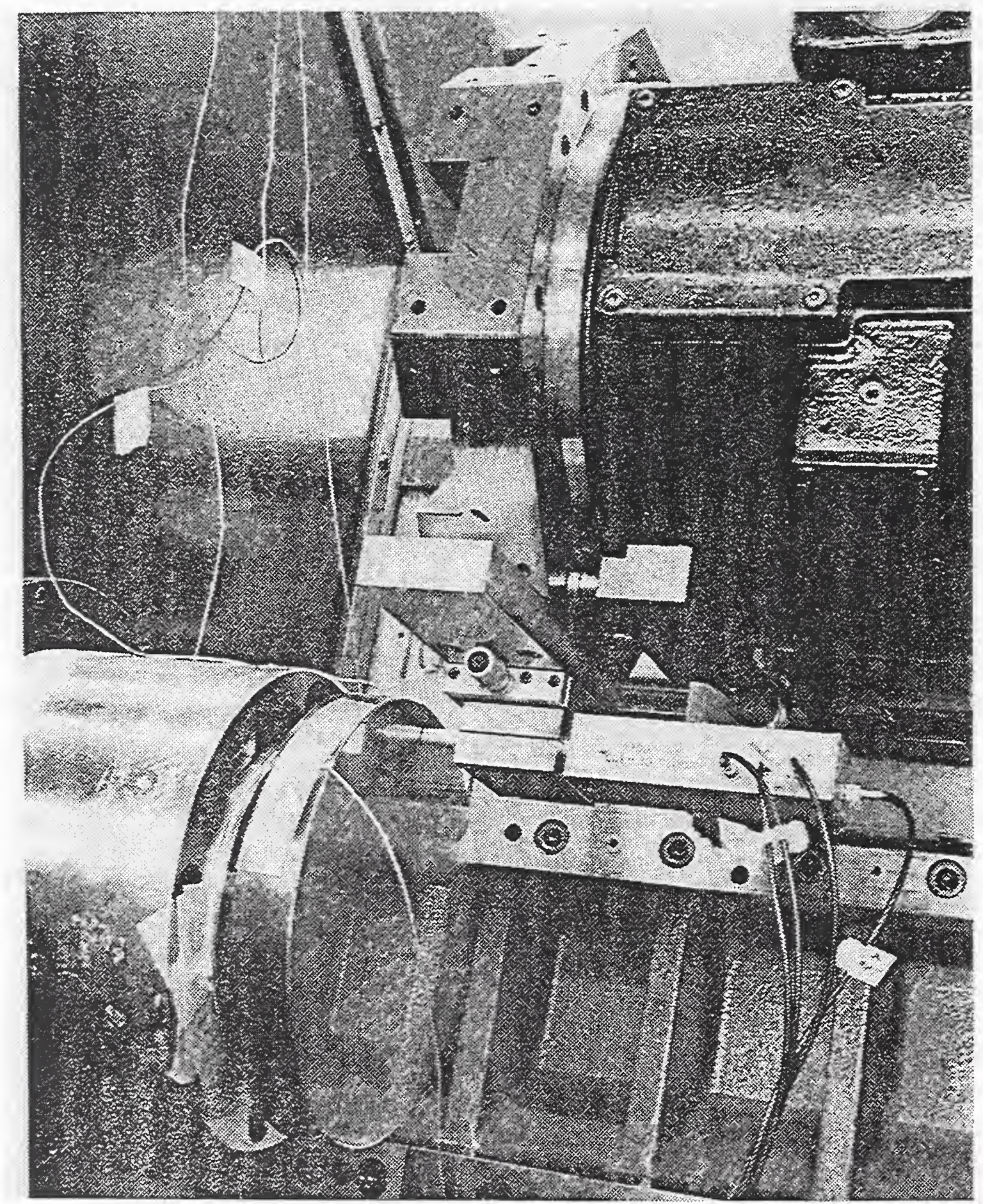

Figure 3.11 The set-up for the $\mathrm{z}$ straightness of the $\mathrm{x}$ motion and the orthogonality measurements 
(a) First set of measurements $\mathrm{m}^{1}(\mathrm{x})$

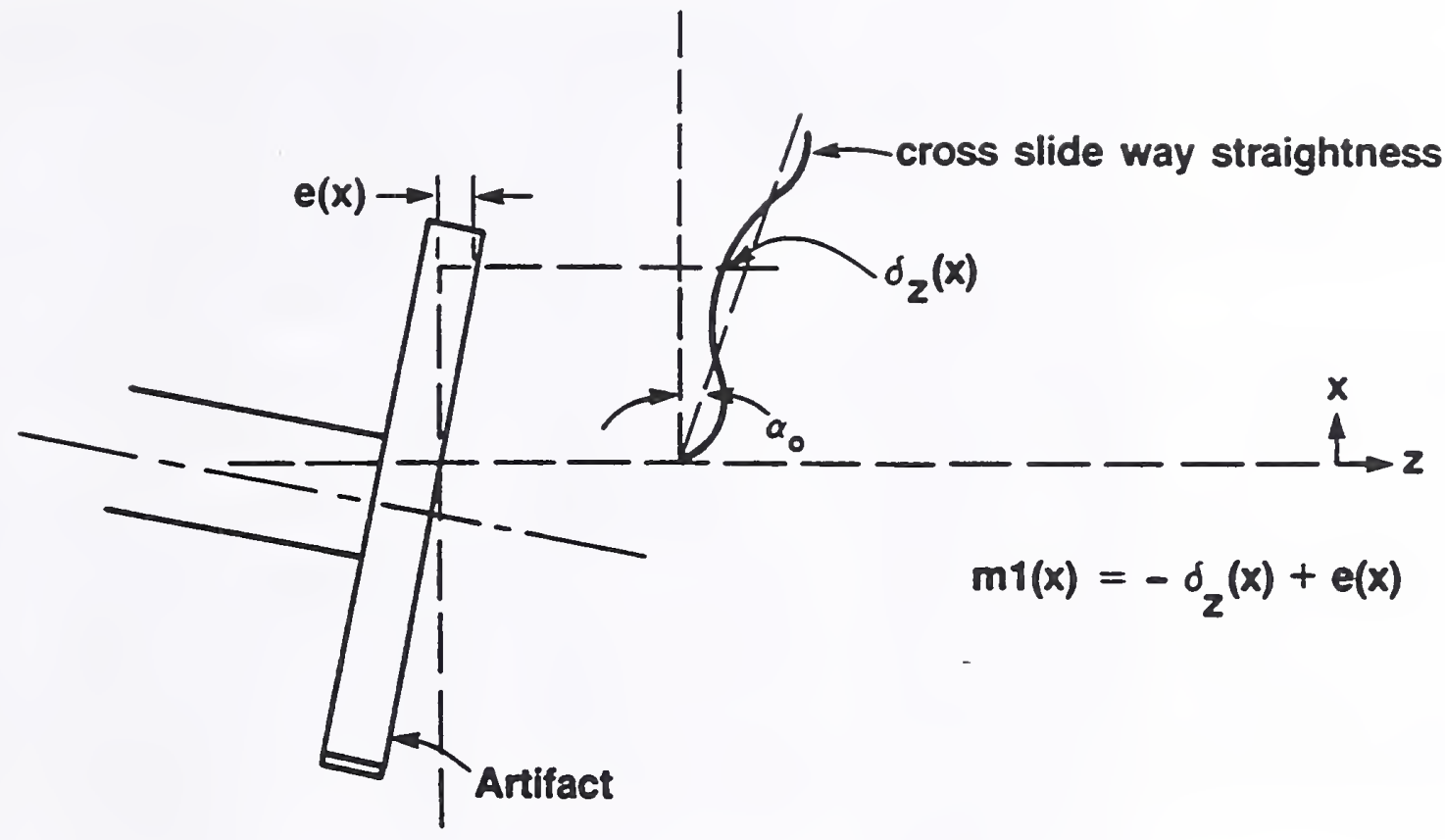

(b) Second set of measurements $\mathrm{m} 2(x)$ after spindle rotated $180^{\circ}$

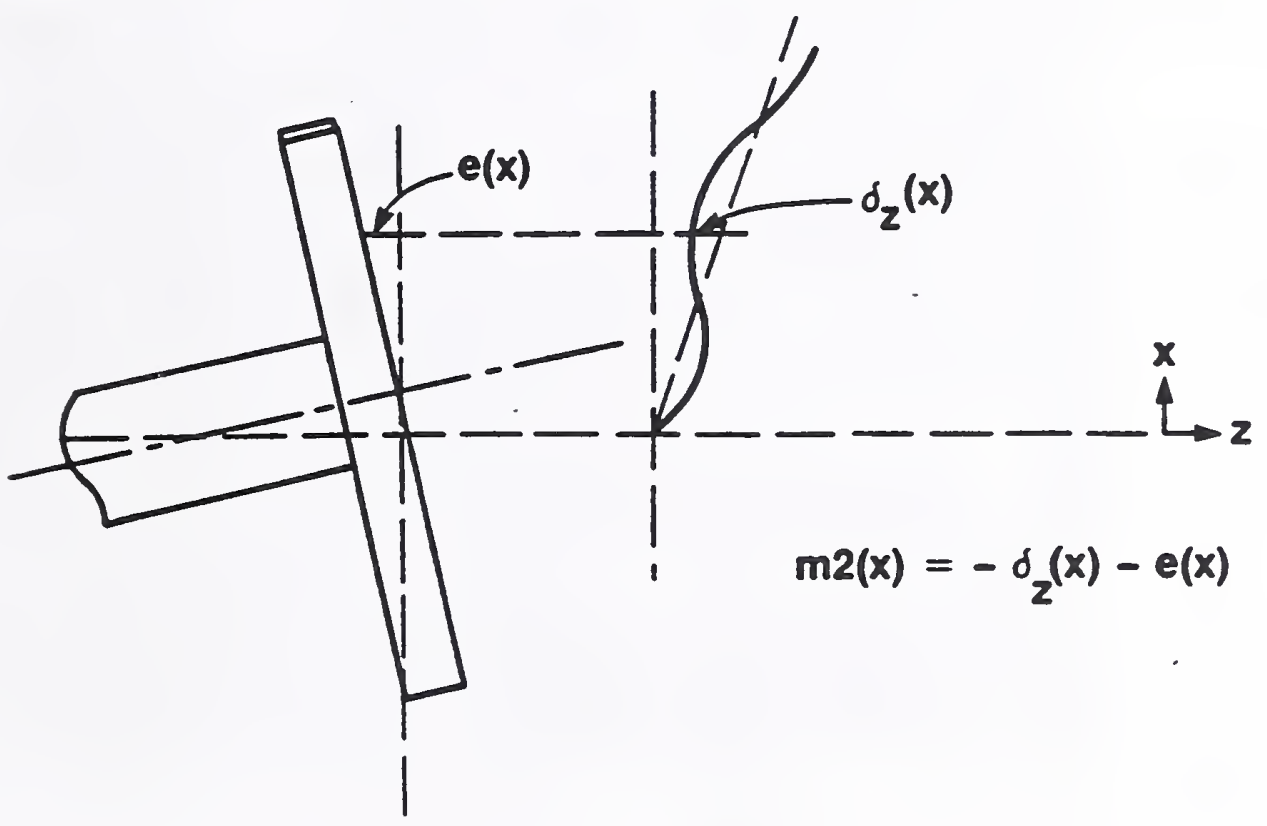

Figure 3.12 The schematic for calculating the $z$ straightness of the $x$ motion 
The orthogonality is calculated as the slope of the best-fit line from the data obtained by Equation 3.8. $\mathrm{X}$-axis squareness with respect to the spindle axis can also be measured by an alternate technique: a part is faced and then scanned using a probe mounted such that it traverses the opposite radius than the cutting tool for the same $\mathrm{x}$-motion as the tool. Twice the squareness error is measured by this technique.

\subsection{Spindle Thermal Drift}

Thermal drift is defined, in the ANSI Axis of Rotation Standard, as "a changing distance between two objects, associated with a changing temperature distribution within the structural loop due to internal and external sources" [11]. As in the case of spindle error motions, three components of spindle thermal drift are critical to the overall performance of the machine tool. They are, axial thermal drift, which is the displacement of the spindle along the $Z$ axis, radial thermal drift, which is the displacement perpendicular to the $\mathrm{Z}$ axis in the sensitive direction, and tilt thermal drift, which is the rotation of the spindle in the $\mathrm{X}-\mathrm{Z}$ plane of the machine.

The equipment used for the measurements consists of the capacitance probes, precision ground test arbors. Figure 3.13 shows the set up for the radial and the tilt drift measurements, and Figure 3.14 shows the set-up for the axial drift measurements.

The conventional way to investigate drift characteristics is to find the changes in the position and orientation of the spindle with time. Using time as an independent variable in the relations complicates the modeling for the prediction of the drift, due to the fact that this approach requires the time history of the operations. Therefore, correlations should be found depending only on currently measured parameters during the operation, such as spindle speed and temperatures of various locations around the spindle.

In order to find the locations at which the temperature changes represent the spindle drift, temperatures are monitored around the headstock during the spindle drift measurements. The locations at which the temperatures are monitored are the spindle bearings on both ends, the spindle face, the four corners of the headstock base, a location on the bed between the 


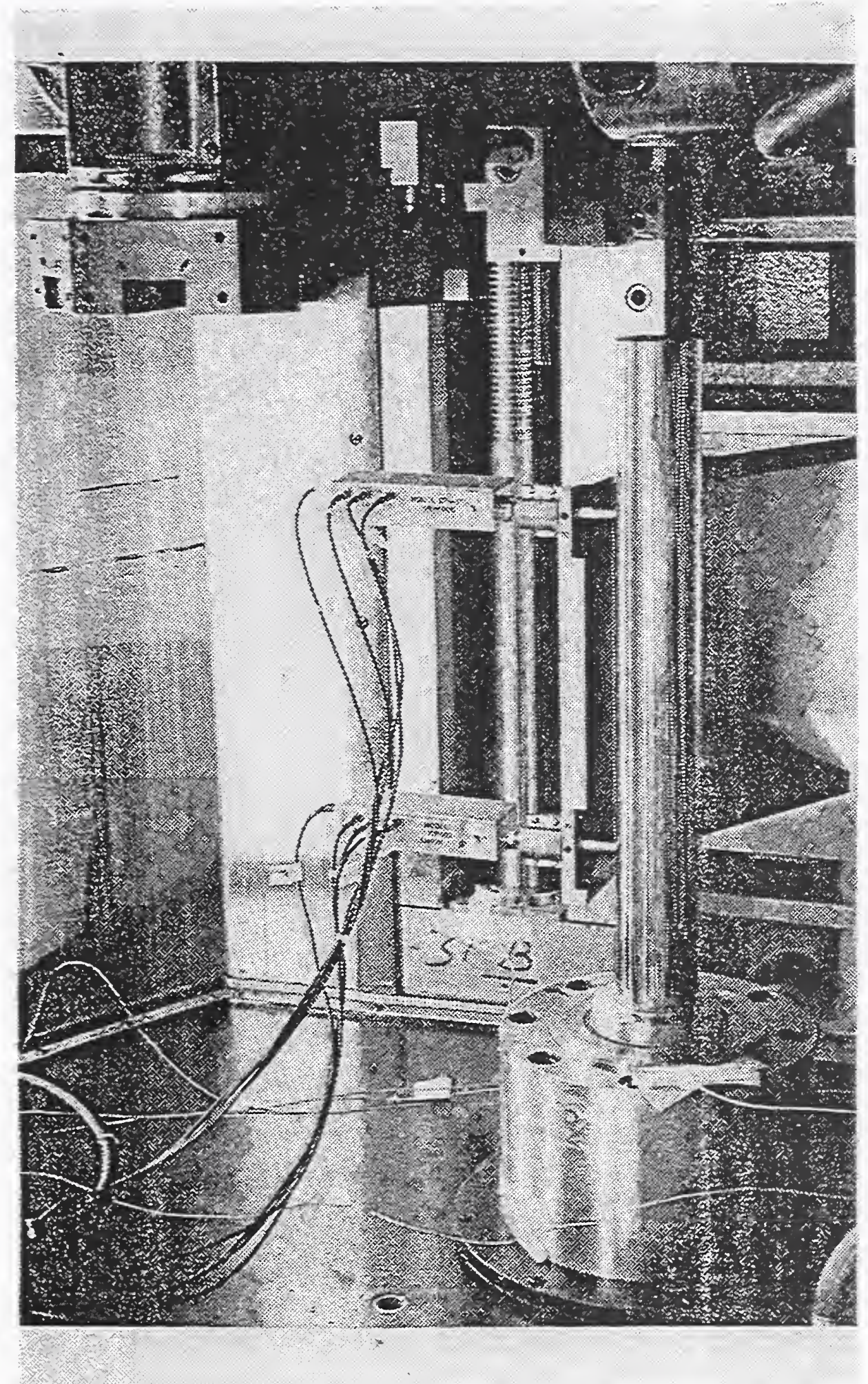

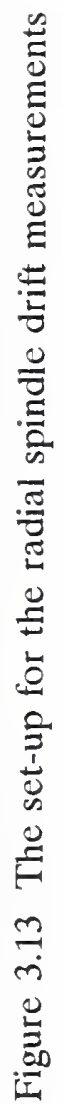




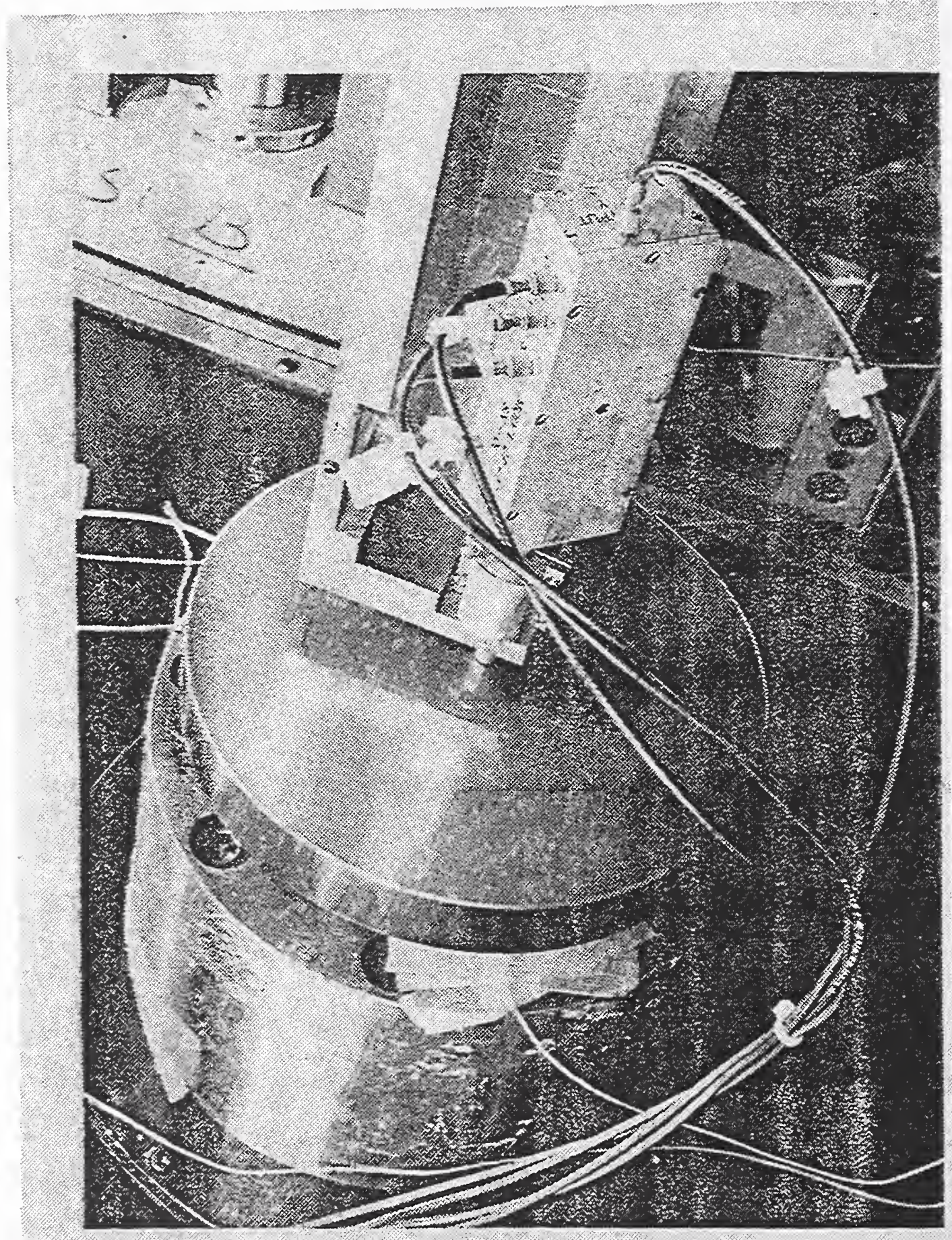

苛 
headstock and the fixture, ambient air, and the probe fixture. These locations are shown in Figure 3.15.

During a typical measurement cycle, the spindle is run at a constant speed for 8 hours, and every 10 minutes, temperature and probe outputs are sampled. In order to eliminate test arbor roundness error and the fundamental spindle error motion, the probe reading is sampled at a particular angular position of the spindle. This is achieved by an optical encoder coupled with the test arbor and interfaced to the system. After 8 hours of running time, the machine is turned off and allowed to cool down overnight, while the temperature and the probe sampling is continued. This procedure is repeated for different spindle speeds, and for both radial and axial directions. The amount of tilt drift at any particular time is obtained by dividing the difference between the two radial displacements measured by the two probes by the distance between the two probes.

\subsection{Data Analysis}

After data have been collected for each machine error component over the operating temperature range and as a function of position and direction of travel, an empirical model is derived for each error component. The collection of these models will be the geometric-thermal (G-T) model for the machine tool. The first step for each error component is to determine the correlation of the error with each of the temperatures that have been measured. The temperatures with the highest correlation coefficient and significant temperature variation are selected as candidates for each model.

A multivariable regression is performed between the error data, axis position, and the selected correlated temperatures. It is likely to be necessary to segment the data depending on the direction of travel due to hysteresis. It may also be necessary to segment the data into regions of axis position. Various types of factors must be considered in the fits to arrive at acceptably low values of standard deviation. Typically, quadratic functions of position are sufficient. Firstorder functions of one or two temperatures, including temperature differences may be required, as well as terms which are a product of temperature and position. Thus, at least one empirical model for each error component must be generated. 


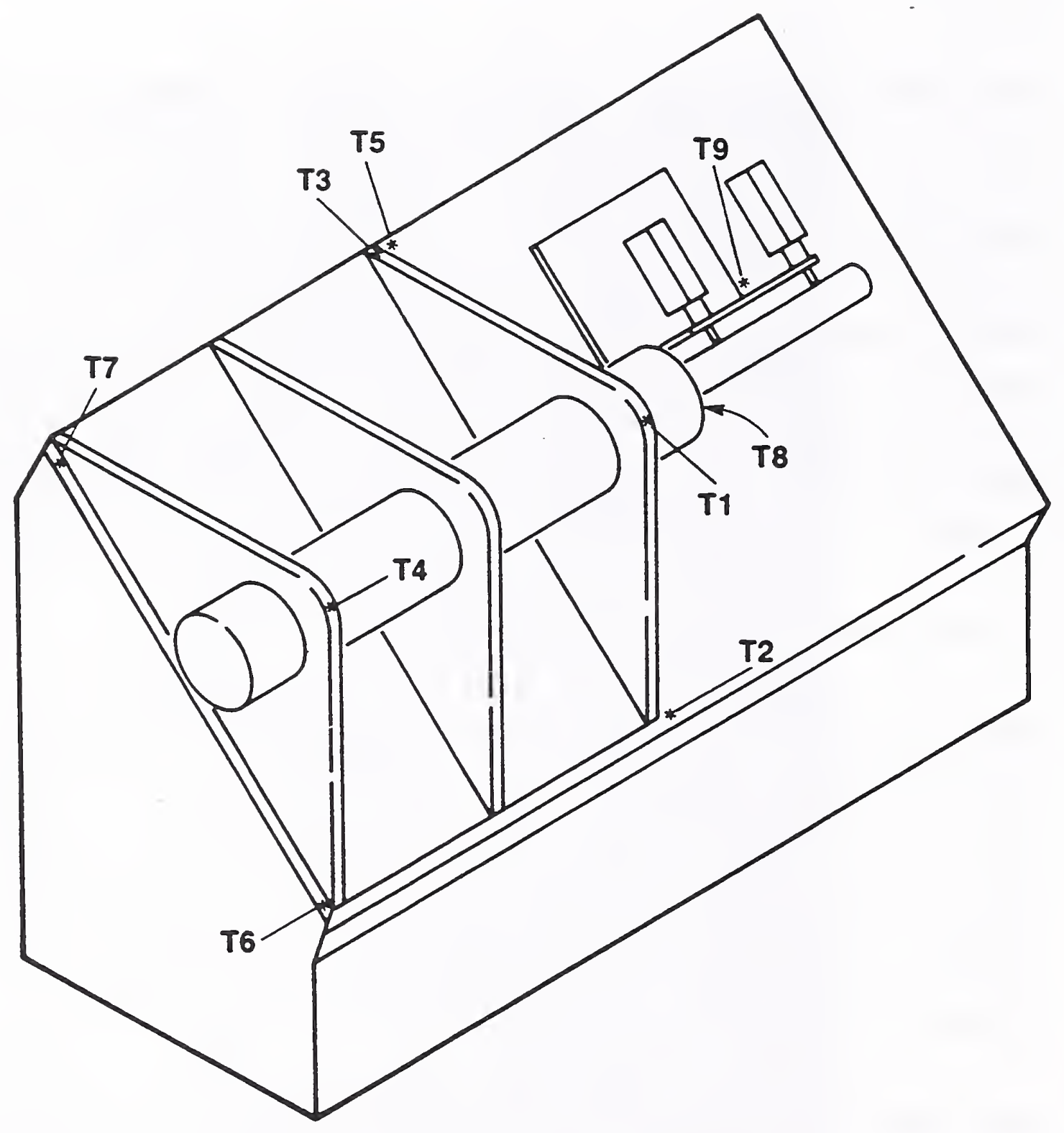

Figure 3.15 Thermocouple locations during the spindle drift measurements 
All of these equations in the G-T model must be evaluated, in real time as a function of axis positions and current temperature readings, to obtain the error components in equations 2.24, 2.25, and 2.26. Solving these equations gives the real-time components of the position error vector. The inverse of these components must be inserted into the machine-tool controller's position loop calculation to compensate for the predicted error. An example of the measurement set-ups, procedures, data analyses and results from measuring nine errors of a turning machine is given in [9].

The measurement and analysis metrology described in this section has been implemented for turning machines. Similar measurement techniques are also applicable to machining centers. In fact, the recently published ANSI/ASME B5.54 Standard describes the measurement techniques required to characterize the performance of machining centers [13]. Following the guidelines provided in this standard, and the analysis and modeling procedures described in this document, a machine tool user or builder should be able to implement various degrees error compensation into existing machine tools. 


\section{Implementing Error Compensation}

\subsection{Potential Problems Limiting Implementation}

In a typical machine tool, the axis' servo motor drives the leadscrew, which in turn moves the machine slide based on the error signal derived from the position command, the position feedback and the velocity feedback signals. The position feedback signal is determined by a position sensor such as a linear glass scale, a rotary encoder, or a rotary resolver. A typical CNC machine-tool controller calculates the position command value at each servo cycle, compares it with the position feedback and provides an error signal as a result of this comparison to drive the servo motor. A real-time error compensation system is an attachment to the controller which inserts an error correction signals into the position servo loop.

After the individual error components have been calculated (as a function of axes' positions and machine temperatures) and combined to find the resultant error components for each axis at the tool-tip (equ. 2.24, 2.25, 2.26), the problem becomes "how to implement the correction" which is, of course, the same magnitude and opposite direction of the error. For example on a two-axis turning machine, the 11 significant errors might combine and require an $\mathrm{X}$-axis

correction $\mathrm{C}_{\mathrm{X}}\left(\mathrm{X}, \mathrm{Z}, \mathrm{T}_{\mathrm{k}, 1, \ldots}\right)=+36 \mu \mathrm{m}$ and a $\mathrm{Z}$-axis correction $\mathrm{C}_{\mathrm{Z}}\left(\mathrm{X}, \mathrm{Z}, \mathrm{T}_{\mathrm{m}, \mathrm{n} . . .}\right)=-25 \mu \mathrm{m}$, where $\mathrm{X}$ and $\mathrm{Z}$ are axis positions and $\mathrm{T}_{\mathrm{k}}$ is the temperature at location $\mathrm{k}$. Note that each error component is a function of both axes' positions and selected temperatures which are not the same for each axis. So how can the correction values be used to modify the tool path to implement the compensation?

In general, for existing commercial machine-tool controllers (MTCs), inserting the corrections is difficult. MTCs are not designed to facilitate error compensation. In a new controller design, the manufacturer could readily include the computation and correction of the position loops required. Changing the axis position values in NC-part programs to alter the tool path is possible, but not attractive. Lead-screw compensation tables are fixed corrections as a function of the axis' own position, i.e., $\mathrm{C}_{\mathrm{x}}(\mathrm{X})$. Recent $\mathrm{MTC}$ and motion controller designs have separate compensation tables that allow cross-axis compensation, e.g., $C_{\bar{x}}(Z)$. These tables would have to be rewritten every few minutes with new values that are a function of the current machine temperature to implement thermal compensation. This may be feasible for some controllers. 
Some MTCs have the capability of inserting a zero or "origin" offset. It has been reported in unpublished work that real-time error compensation using the method has been implemented on at least two models of MTCs. Machine specific software modifications, perhaps to the executive software which is not readily available to the user, is required.

\subsubsection{Case Study Using Hardware Modifications}

The NIST Manufacturing Engineering Laboratory has previously implemented error compensation on several machine tools. Two of them are a $15-\mathrm{kW}(20-\mathrm{hp})$ vertical machining center (VMC) and a 12.5-kW (16.8-hp) turning center (TC). The feedback elements for the VMC are linear glass scales, and the output signals are conditioned to appear as resolver signals (two sine wave signals that change in relative phase by 360 degrees for one "rotation") shown in fig. 4.1. The position information is obtained by "piggy-backing" the counter-latches which contain the raw-count axis position in the controller. This technique was possible only because the manufacturer provided the physical location and logical address of the registers holding the position counts. The counts were simultaneously and independently latched and sent by an 8051 family microcomputer to a minicomputer for off-line correction calculation. In these calculations, machine-tool geometric errors of the X-axis, as well as thermal growth of the spindle head in $\mathrm{X}$ and $\mathrm{Y}$ directions were taken into account. The spindle growth was measured in both the $\mathrm{X}$ and $\mathrm{Y}$ directions by two linear variable differential transformers (LVDTs) mounted at the end of the machine-tool table. Periodically, these LVDTs were moved to a reference position where readings of displacement of the spindle head were taken. These displacement readings, along with the axes' positions, were used to compute position corrections, which were sent back from the minicomputer to digital-to-analog (D/A) converters. These D/A converters feed the reference inputs of analog comparators in the MTC that convert the "resolver" output sine waves to square waves. Changing the reference voltage produces a phase shift between the sine wave and the square wave which makes the controller stop the axis at a slightly different position than without the correction. The amount of control available allows about $\pm 0.1 \mathrm{~mm}$ ( \pm 3.5 milliinches) change in the axis positions, which is invisible to the controller. The resolution of the correction is limited by the number of bits of the $D / A$, since the correction is analog. For example, an 8-bit D/A converter will resolve the correction signal down to 1 part in 256 , or about $0.7 \mu \mathrm{m}$ ( $30 \mu \mathrm{in})$. Only the $\mathrm{X}$ and $\mathrm{Y}$ axes of this machine were compensated since the primary application requiring the compensation was drilling, which is not sensitive to Z-axis 
errors.

\subsubsection{Case Study Using Software Modifications}

The turning center, which has a controller to compensate for error without any hardware modifications, is shown in fig. 4.2 [14]. The machine has resolver feedback, but since the position correction is done entirely in software, this is immaterial. An 8086 based single-board microcomputer, running at $8 \mathrm{MHz}$ with a high-speed version of the 8087 math coprocessor, was
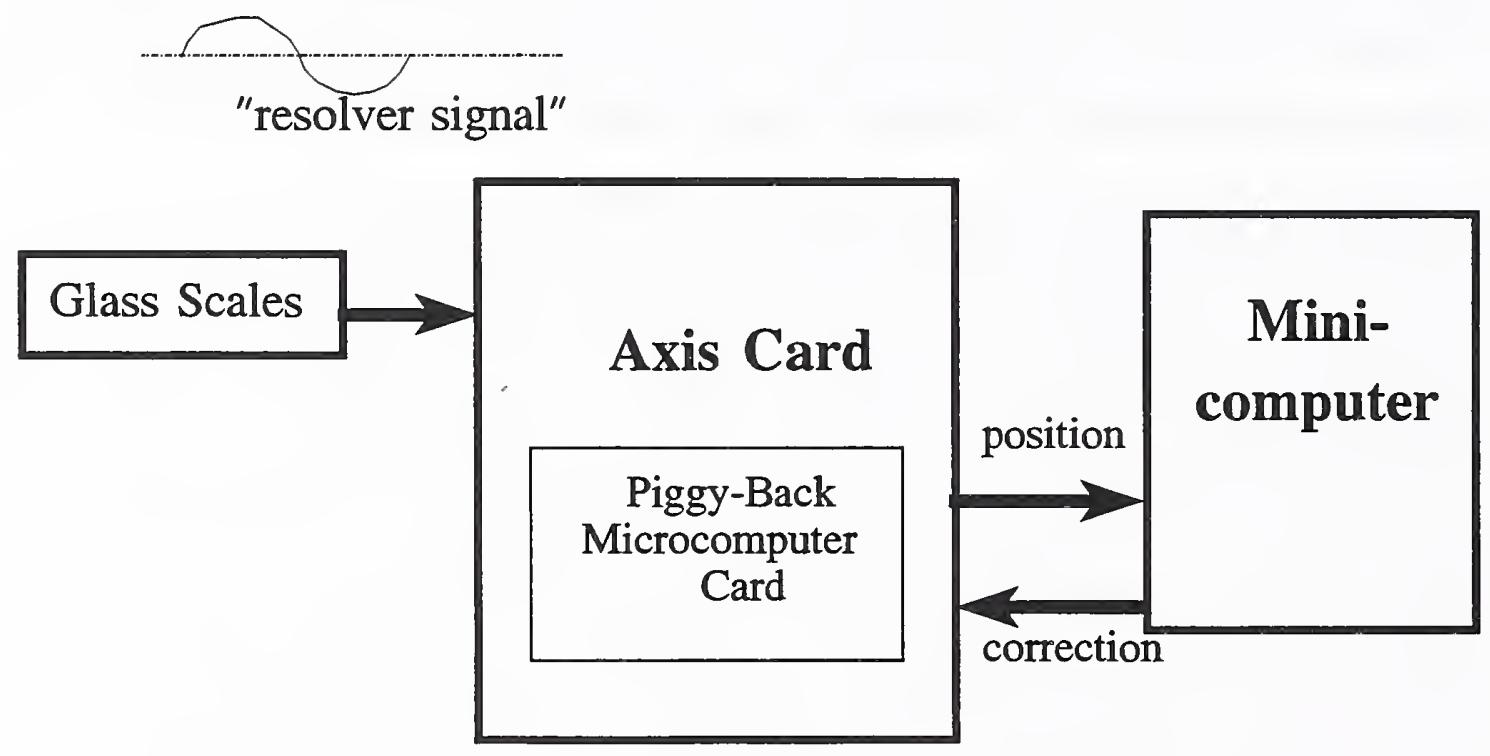

Figure 4.1 Hardware specific error compensation 


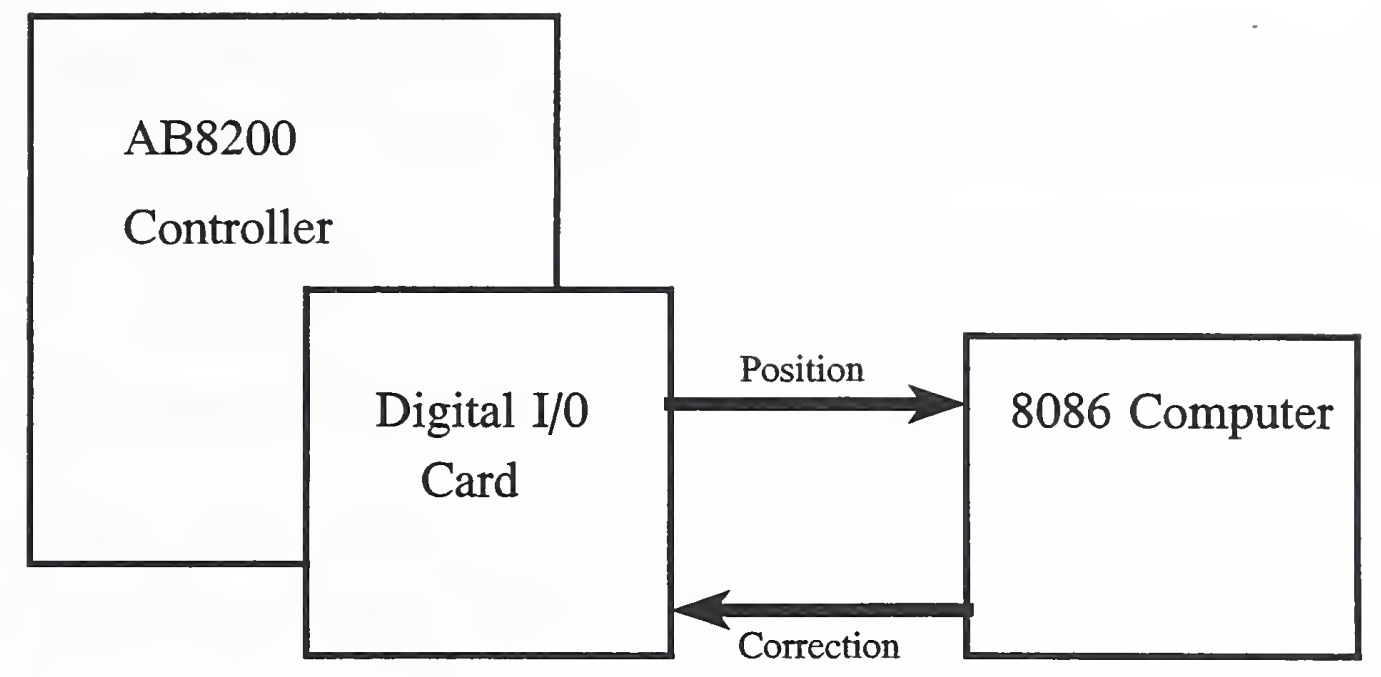

Figure 4.2 Software specific error compensation

used to calculate the corrections. The MTC's Programmable Application Logic (ladder network) software was modified to output the axes' positions and input the axes' corrections. Digital Input/Output (I/O) interface boards in the MTC provide axes' positions to the microcomputer. The computed correction was fed back into the MTC by modifying the contents of the "following error" register which holds the difference between the programmed position and the position indicated from the processed resolver signals. The axis reaches the desired position when the following error is zero, and the modification of this following error causes the machine to be at a different position than it would without the correction. The resolution of the correction is the resolution of the machine-tool controller. A new correction is calculated and inserted at every control cycle of $20 \mathrm{~ms}$. This correction rate approaches the computational speed of the 8086 computer used for this application. Both the $\mathrm{X}$ and $\mathrm{Z}$ axes of this machine have error compensation. Dimensional accuracy improvements on the order of 10 to 20 times, in diameter and length, were achieved over the cold-to-operating temperature range, and 
straightness was improved by a factor of 4 to 5 over a 70 -mm length.

The previous two cases are very MTC specific--one used hardware, the other software. The compensation systems used are only a applicable to the specific MTC designs.

\subsection{A Generic Approach}

A generic method of implementing compensation on any MTC with "encoder-type" position feedback has been developed. We have inserted a black box, called the Real-Time Error Corrector (RTEC) [15], between the position feedback elements of the axes and the MTC as shown in figure 4.3. This device independently and simultaneously counts the signals from the feedback element to produce the machine position and includes an interface to send this position data to a PC-type computer which performs the error calculation. The device allows real-time correction of axis' positions without intrusion into the MTC and in a way that is invisible to the MTC and the user. A minimum of hardware and software is MTC specific. The position feedback signals are modified to make the machine axes go to slightly different physical positions (as commanded by the PC) than they would without the corrections applied.

\subsubsection{RTEC Operating Principles}

Encoder-type signals, shown in figure 4.4, have two square-wave output signals (A and $\mathrm{B}$ ) that are displaced in phase by $90^{\circ}$ (one-quarter cycle). These signals have the same period and a fifty-percent duty cycle. The signal which leads in phase changes with the direction of axis motion. An additional signal, called the reference or index, produces one pulse per revolution of the encoder. This signal, which is used when the axis is referenced, will not be modified by the RTEC and is not shown in the figure. The frequency of the square waves is a function of the number of lines per revolution in the encoder (perhaps 4096) and the rpm of the encoder. Therefore, at rest, the two signals are static and either may be at high or low logic levels. Figure 4.4 shows the $\mathrm{A}$ and $\mathrm{B}$ signals with the axis moving in the positive direction (phase $\mathrm{A}$ leads phase B). Each cycle of the square waves corresponds to a linear distance of axis movement which depends on the number of lines per revolution in the encoder, the gear ratio between encoder and the ball screw and the ball-screw pitch. The encoder-type version of the

RTEC must contain circuitry to count the encoder cycles and determine which direction the axis 
is moving in order to know whether to add or subtract counts. This circuitry gives more resolution, by a factor of 4 . To make the machine axis stop sooner, by the distance corresponding to one square-wave cycle, the RTEC must insert extra pulses with the correct phase relationship (A leading B) as shown in figure 4.4. Likewise, to make the axis go one cycle further, the RTEC must subtract one pair of pulses as in figure 4.4, or alternatively, insert a pair of pulses with the phase B leading A. Note that the resolution of the correction is the displacement corresponding to one square-wave cycle. This correction resolution is four times coarser than the axis position resolution.

Alternative designs which overcome some of the limitations of the original design and allow finer correction resolution are currently being implemented [16].

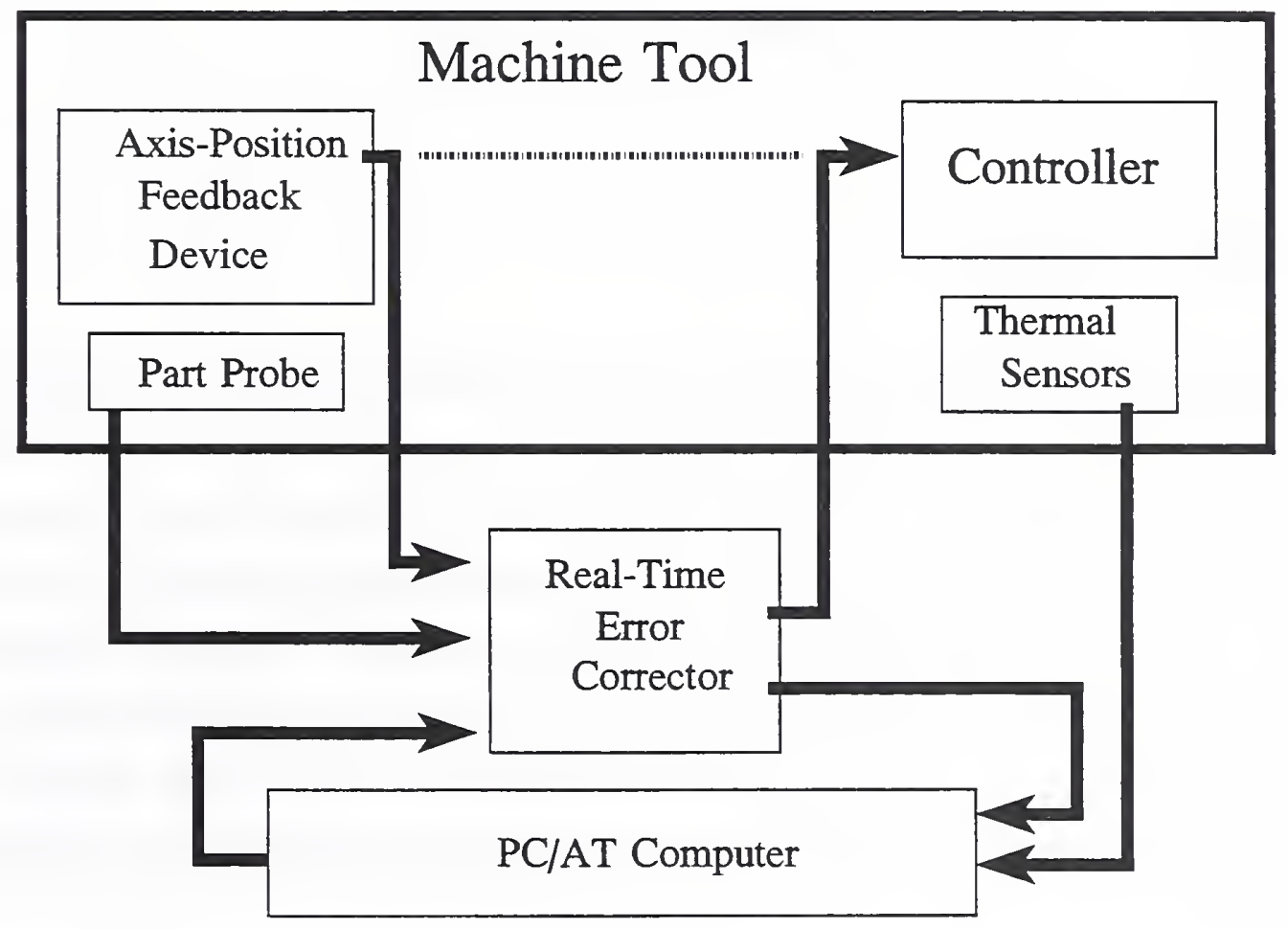

Figure 4.3 System Overview 


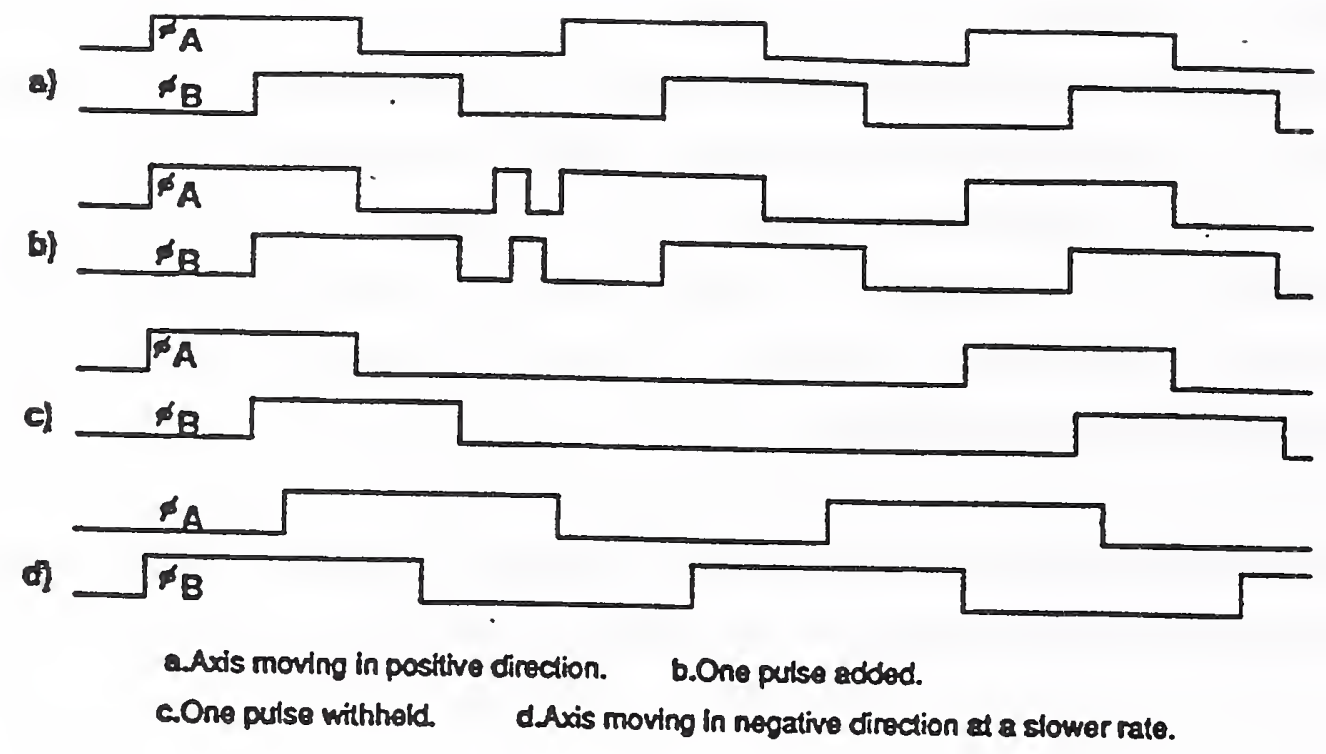

Figure 4.4 Encoder Signals

\subsubsection{The Way of the Future}

New designs of MTCs on the market have multiprocessors with enormous computational power compared to MTCs of the 1980's. It is not uncommon for a front-end user interface to be a 286,386 , or even 486 based PC. In addition, the axes' servo calculations may be performed by a Reduced Instruction Set Computer (RISC) or one or more Digital Signal Processor (DSP). For three axes, 32-bit servo loop calculations in under $10 \mu$ s is common. With these designs, the error computation could readily be done by the $\mathrm{PC}$ as a background task running under a multitasking operating system. Correction values for each axis can be passed to the processor implementing the position servo loop. Yet, most new MTCs are not designed to perform compensation.

Recently, two commercial machine tools have been shown with partial (not all axes or not a function of axes positions) error compensation. One uses a proprietary MTC made by the machine-tool manufacturer, the second uses an MTC from a company that has relatively recently entered the controller market. Some "open architecture" MTCs may allow the 
required changes to be made by the user. Several MTCs could readily include error compensation with software modifications by the manufacturer. Evolution is on the horizon. With these designs, the error computation could readily be done by the PC as a background task running under a multitasking operating system. Correction values for each axis can be passed to the processor implementing the position servo loop.

A recent project at the National Institute of Standards and Technology is developing an Enhanced Machine-Tool Controller (EMC). This work will demonstrate the benefits of an "open architecture" machine-tool controller with interfaces to incorporate inputs from sensors to monitor the machine and machining process. The EMC will have the ability to calculate the predicted error components from a geometric-thermal model and implement the corrections. The EMC will eliminate the barriers to implementing error compensation which are present in nearly all commercial machine-tool controllers today. 


\section{Summary}

Some guidance has been presented for implementing error compensation for geometric and thermally induced errors of a machine tool. Unfortunately the state-of-the-art does not allow a "cook book" to be written. Successful implementation of error compensation requires a thorough understanding of the error components for the particular machine tool being compensated, the measurement techniques used to characterize the errors, the mathematical techniques used to model each error component, and the ability to develop a mathematical model for the particular machine tool's errors. Last, but not least, a method of implementing the compensation for the predicted resultant error components at the tool-tip must be available.

We have presented the development of a mathematical model for the errors of a two-axis turning machine. Next, a procedure is described for characterizing the error components of a turning machine. An example of actual error component measurements of a two-axis turning machine, using some alternate measurement techniques, is given in [9]. Last, some of the known techniques for implementing the predicted error compensation on existing machine-tool controllers is presented. Hopefully, new generation controllers will include the software and/or hardware to facilitate error compensation. The fact that two machine-tool manufacturers have introduced models, using different controllers, with at least partial geometric-thermal error compensation is an indication that the technique "has arrived." 


\section{References}

1. M.A. Donmez, et al., "A General Methodology for Machine Tool Accuracy Enhancement by Error Compensation," Precision Engineering, vol.4, 1986.

2. M.A. Donmez, et al., "A Generalized Mathematical Model for Machine Tool Errors," in Modeling, Sensing, and Control of Manufacturing Processes, Bk. No. H00370, K. Srinivasan, D.L.E. Hardt, and R. Komanduri, eds. (ASME, New York, 1987)

3. R.P. Paul, Robot Manipulators: Mathematics, Programming, and Control, The MIT Press, 1981.

4. C.S.G. Lee, "Robot Arm Kinematics," Tutorial on Robotics, IEEE Computer Society, Washington, DC, 1983.

5. J. Tlusty, "Techniques for Testing Accuracy of NC Machine Tools," Proc. of 12th MTDR Conference, University of Manchester, Manchester, U.K., 1971.

6. J. Denavit and R.S. Hartenberg, "A Kinematic Notation for Lower Pair Mechanisms Based on Matrices," Journal of Applied Mechanics, June 1955.

7. W.T. Estler, "High-Accuracy Displacement Interferometry in Air," Applied Optics, vol. 24, No. 6, 1985.

8. Hewlett Packard Laser Interferometer Model 5528 User Manual

9. K. Harper, "Determination of the Geometric Thermal Errors of a Turning Machine," (to be published).

10. M.A. Donmez, A General Methodology for Machine Tool Accuracy Enhancement-Theory, Application and Implementation, Ph.D. Dissertation, Purdue University, August 1985. 
11. R. Donaldson, "A Simple Method for Separating Spindle Error from Test Ball Roundness Error," Annals of CIRP, vol. 21, 1972.

12. Axes of Rotation, ANSI Standard, B89.3.4-1983.

13. Methods for Performance Evaluation of Computer Numerically Controlled Machining Centers, ANSI Standard, B5.54-1992.

14. M.A. Donmez, et al., "A Real-Time Error Compensation System for a Computerized Numerical Control Turning Center," Proc. of IEEE International Conf. on Robotics and Automation, (IEEE Computer Soc., Washington, D.C., 1986)

15. R.J. Gavin and K.W. Yee, Implementing Fast Part Probing and Error Compensation on Machine Tools, NISTIR 4447, National Institute of Standards and Technology, Gaithersburg, MD, 1990.

16. K. W. Yee, Alternative Designs of a Real-Time Error Corrector for Machine Tools with "Encoder" Position Feedback, NISTIR 4832, National Institute of Standards and Technology, Gaithersburg, MD, 1992. 

